\title{
Gratings in Structured Optical Fibres
}

\section{John Canning, ${ }^{1}$ Nathaniel Groothoff, ${ }^{1}$ Kevin Cook,, ${ }^{1}$ Cicero Martelli,, ${ }^{1,2}$ Alexandre Pohl,, 3 John Holdsworth, ${ }^{1,4}$ Somnath Bandyopadhyay, ${ }^{1,5}$ and Michael Stevenson ${ }^{1}$}

${ }^{1}$ Interdisciplinary Photonics Laboratories, School of Chemistry, University of Sydney, Sydney, NSW 2006, Australia

${ }^{2}$ Mechanical Engineering Department, Pontifical Catholic University of Rio de Janeiro-PUC-Rio, Rio de Janeiro 22453-900, Brazil

${ }^{3}$ Federal University of Technology-Paraná, Curitiba 80230-901, Brazil

${ }^{4}$ School of Mathematical and Physical Sciences, Faculty of Science and Information Technology, Newcastle University, Newcastle, NSW 2308, Australia

${ }^{5}$ Central Glass and Ceramic Research Institute, Kolkata-700032, India

Correspondence should be addressed to John Canning, j.canning@usyd.edu.au

Received 10 June 2008; Accepted 18 August 2008

Recommended by Stavros Pissadakis

\begin{abstract}
Grating writing in structured optical fibres and their properties and applications are reviewed. To date, most gratings have been written in a straightforward manner into structured fibres containing a photosensitive germanosilicate step-index core. However, gratings have also been written directly into single material, structured silica fibres and into air-clad cores using two and higherphoton processes with both UV and near IR pulsed (nanosecond-femtosecond) light. Given the intrinsic-added functionality possible within a structured optical fibre, structured fibre gratings offer further capabilities for sensors, diagnostics, lasers, and devices.
\end{abstract}

Copyright (c) 2008 John Canning et al. This is an open access article distributed under the Creative Commons Attribution License, which permits unrestricted use, distribution, and reproduction in any medium, provided the original work is properly cited.

\section{Introduction}

Structured optical fibres such as suspended core fibres [1], photonic crystal fibres [2], and Fresnel fibres [3] introduce a new degree of functionality hitherto not possible. For example, the composite properties of a structured optical fibre can be tailored by adding materials into the holes. This opens up a range of new possibilities, from zero temperature-dependent fibres and components, including gratings [4], to advanced functionality possible by superposing multiple properties; an example is an optical fibre white light source made by superposing the properties of individual dye molecules that are spatially separated to avoid quenching [5]. Access to the evanescent field over long lengths had enabled direct evanescent field spectroscopy of silica interfaces, revealing new bands not possible without resonant techniques [6].

Adding spectral selectivity to extend with similar ease the possible applications using laser written components such as fibre Bragg gratings demands a new level of engineering in grating writing since structured fibres introduce several challenges to grating writing not present in conventional fibres. These include high levels of scattered light arising from multiple interface reflections and, more critically, rotationally variant symmetry. Nevertheless, straightforward conventional single-photon Bragg grating writing has been demonstrated with $244 \mathrm{~nm}, 266 \mathrm{~nm}$ (nanosecond and femtosecond pulsed), $193 \mathrm{~nm}$ and in various structured fibres that contain conventional step index germanosilicate cores [7-12]. Unfortunately, for many applications, the advantages of having a surrounding structured cladding are lost when conventional step cores are inserted. Further in many applications, such as $\mathrm{Er}^{3+} / \mathrm{Yb}^{3+}$-codoped lasers, germanium present in the core is undesirable. Hydrogen loading is one approach for writing gratings using $193 \mathrm{~nm}$ in nongermanosilicate structured optical fibres [9]. Alternatively, two-photon absorption directly into the core has also been used to write gratings in pure silica core conventional step index fibres with a fluorinated cladding [13] and in all-silica, single material-structured fibres including photonic crystal fibre [14] and Fresnel fibres [15]. The generic nature of two-photon excitation 
allowed gratings to be written into structured fibres for laser applications both as distributed Bragg reflector (DBR) elements and as phase-shifted distributed feedback (DFB) cavities [16-18]. The fibre in these cases was an $\mathrm{Er}^{3+}$ doped aluminosilicate core photonic crystal fibre. Using two-photon absorption into the band edge avoids the need for hydrogen loading. The specific need of fibre lasers has also seen the inscription of both single-photon [19] and multiple-photon Bragg gratings [20] within Ybdoped core air-clad nanostructured optical fibres specifically fabricated for high-power fibre lasers. This work has been complemented in photonic crystal fibres by higher-exponent processes made possible using femtosecond lasers [21]. An updated review on photosensitivity in general is given in [22].

Although grating writing has been demonstrated in a range of structured optical fibres, the rotational variance of these fibres, however, leads to variations in the writing intensity across the core from the side during subsequent grating writing, posing serious challenges to reproducibility and predictability. Unlike conventional fibre grating writing, these must be considered if the process is to be brought under control. This surrounding structure leads to scattering, diffractive in the case of regular or quasicrystalline structures, which actually carries information regarding the structure of that fibre-indeed, such a property has been proposed as a simple photonic encryption key [23]. For grating writing, an obvious solution is to fill the holes with index matching fluids which can be subsequently removed [8]. However, for shorter wavelengths such as $193 \mathrm{~nm}$ solutions with appropriate transmission are not readily available. Higherphoton effects, both in the UV and in the near IR, are also unable to access the core without significant absorption and heating within the liquid itself, which can damage the fibre. If the structure is sufficiently removed from the step-index core, this problem can be overcome [20]. Here, we review the progress to date on grating writing within structured optical fibres and discuss some of the challenges and directions to further extending the capability of structured fibres using gratings. We also elaborate some of the unique properties and applications of structured optical fibres using Bragg gratings.

\section{Scattering from the Structured Cladding}

Typical structured optical fibres into which gratings have been successfully inscribed are illustrated in Figure 1. Each serves a purpose, ranging from gas and liquid sensing, biodiagnostics, novel components, and fibre lasers, which can be enhanced with gratings, as outlined in the figure caption. Structured optical fibres include two main types: (1) step-index analoges, where the cladding made up of air holes has an average index lower than that of the core, and (2) diffractive fibres, or Fresnel fibres, which includes Bragg fibres, omniwaveguides, and other photonic bandgap fibres exploiting crystal and quasicrystal symmetry $[2,3,24]$. In practice, structured step-index fibres also have significant diffractive effects particularly as the wavelength becomes shorter [25]. Within these categories, there are even finer details. When light strikes the structured cladding layers, both the light which is able to reach the core and the light which reaches beyond the other side of the fibre are dependent on the regularity of the lattice, hole size, hole number, lattice period, and interstitial hole spacing [26-31]. For example, a normal triangular lattice photonic crystal fibre will have repeating angular structure every 30$60^{\circ}$. Such crystal lattices can also act as lenses which may offset scattered light by focussing the light that gets through into the core, or which at another rotation angle leads to complete defocusing of light out of the core, depending on the parameters of the cladding structure [28].

There are two approaches to studying the scattered light: (1) examine the signature of the scattered light through and beyond the structured lattice in the far field; or (2) study the light reaching the core of the structured optical fibre. Each case is considered briefly.

\subsection{Measuring Scattered Light through the Structured Fibre}

Far-field measurements of scattered light can provide information about the structured cladding, particularly periodicity [23]. Figure 2 shows the experimental setup that was employed to investigate scattered light from the side. A five wavelength tunable HeNe laser is used as a flexible optical source. The beam is directed perpendicularly to the fibre sample and the scattered light is project on a CCD camera (CCD-1) at a distance $d$ from the fibre sample. The beam spot size is at least three times bigger than the fibre external diameter and the intensity is adjusted using a neutral density filter to be the same for all wavelengths in all measurements $(P=0.7 \mu \mathrm{W})$. The fibre sample holder is placed on a rotation stage that controls the fibre angular orientation. An $x y$ stage set is used to position the fibre at the center of the rotation stage. The structured fibre angular position is determined by imaging its end face with an objective lens and projecting the image on a CCD camera (CCD-2) connected to a TV monitor. An example of the interference pattern generated by sending HeNe light at $543 \mathrm{~nm}$ through the side of a fourring-structured optical fibre recorded by CCD-1 is, along with an SEM cross-section of the fibre, also shown. The scattered pattern is easily correlated to the regular lattice structured determined by the four rings.

The number of fringes displayed within a defined angle range is directly proportional to the fibre diameter and can be written as [23]

$$
N=\frac{2 b}{\lambda}\left\{\begin{array}{c}
{\left[\sin \frac{\theta_{2}}{2}+\left(m_{1}^{2}+1-2 m_{1} \cos \frac{\theta_{2}}{2}\right)^{1 / 2}\right]} \\
-\left[\sin \frac{\theta_{1}}{2}+\left(m_{1}^{2}+1-2 m_{1} \cos \frac{\theta_{1}}{2}\right)^{1 / 2}\right]
\end{array}\right\},
$$

where $b$ is the fibre radius, $m_{1}$ is the fibre cladding refractive index, $\theta_{1}=$ incident angle to the $\Gamma \mathrm{K}$ direction, and $\theta_{2}=$ scattered angle. Hence the number of fringes within the angle range is also a function of the laser wavelength. The 


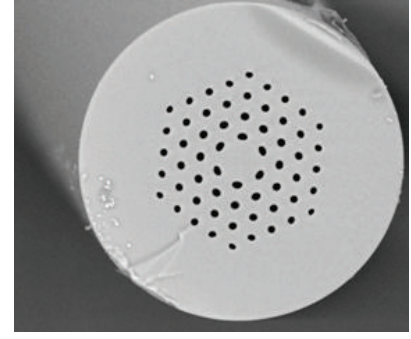

(a)

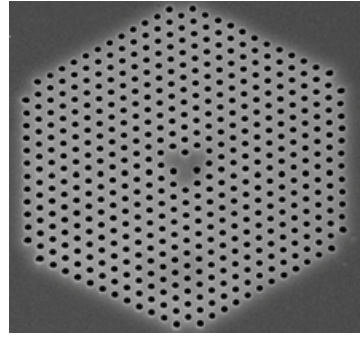

(b)

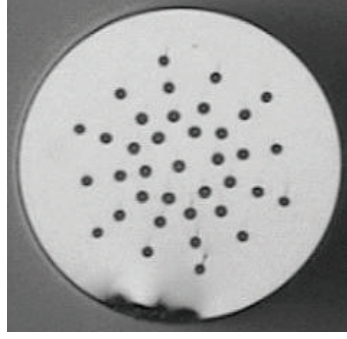

(c)

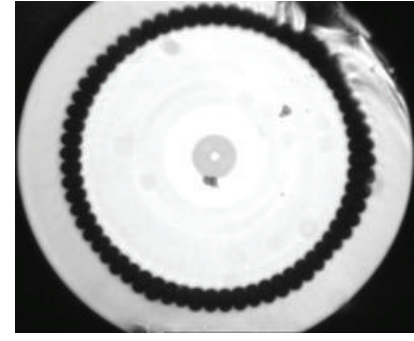

(d)

FIgURE 1: Typical structured optical fibres into which gratings have been written: (a) low-loss $(<4 \mathrm{~dB} / \mathrm{km}) 4$-ring all-silica photonic crystal optical fibre; (b) 12-ring photonic crystal fibre with triangular core and photosensitive, high-NA step-index germanosilicate center; (c) simple Fresnel fibre; and (d) air-clad fibre with $\mathrm{Yb}^{3+}$-doped core.

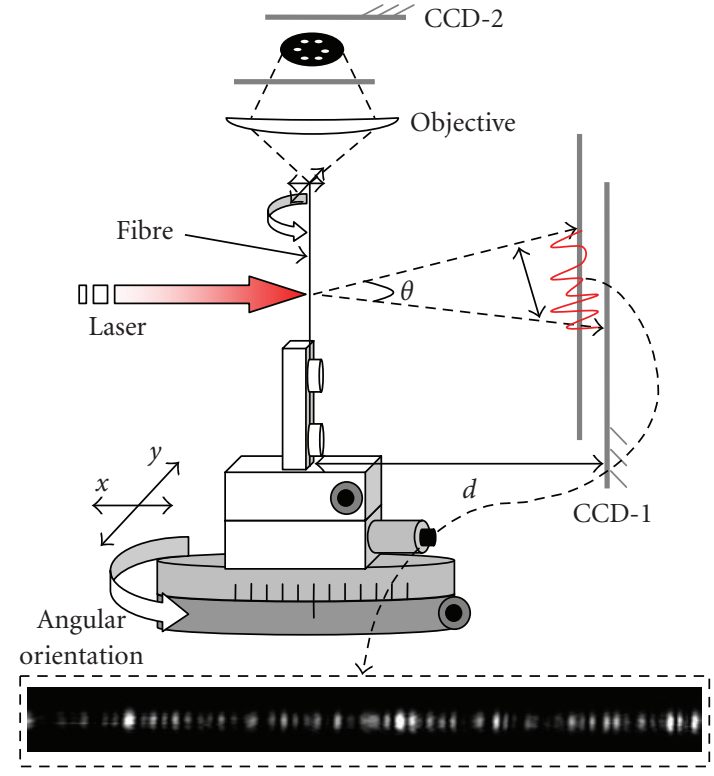

(a)

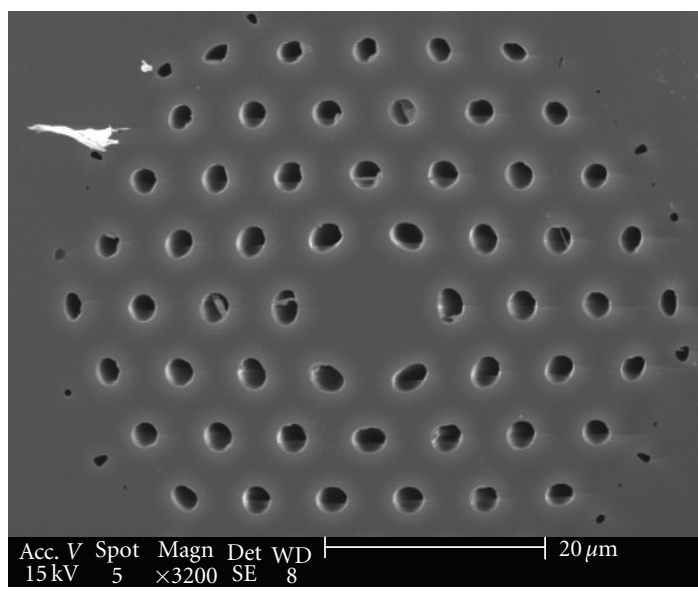

(b)

FIGURE 2: (a) Experimental setup used to produce the interference patterns and example of the recorded pattern obtained from a 4-ring photonic crystal fibre; (b) SEM cross-section of 4-ring, single material (silica) photonic crystal fibre ( $\Phi_{\text {ext diameter }} \sim 100 \mu \mathrm{m}, \phi_{\text {hole }} \sim 4.5 \mu \mathrm{m}$, and $\Lambda \sim 8.5 \mu \mathrm{m})$. More details in [23].

system is calibrated analyzing the fringe pattern of fibre1 (a standard step-idnex fibre which is single mode at $632 \mathrm{~nm}$ ) four different wavelengths $(543,594,604$, and $633 \mathrm{~nm}$ ). Since the fibre diameter was known, the number of fringes was used as an evaluation parameter. The calculated and measured number of fringes is the same and the results presented in Table 1.

The patterns at each wavelength generated with the 4ring-structured fibre similar to that in Figure 1(a) are presented in Figures 3(a)-3(c). The FFT plot of one interference pattern at $543 \mathrm{~nm}$ (Figure 3(b)) shows a broad frequency distribution, consistent with no obvious periodic pattern. The intensity plot at $543 \mathrm{~nm}$ shows a random distribution of peaks (Figure 3(c)). The calculated frequency using the previous equation is consistent with the peak of the broad FFT spectrum and we interpret this to mean that most of the rays reflect at the cladding air interface. The intensity plot
TABle 1: Measured and calculated number of fringes for four wavelengths [23].

\begin{tabular}{ccc}
\hline $\begin{array}{c}\text { Wavelength } \\
(\mathrm{nm})\end{array}$ & $\begin{array}{c}\text { No. of Figures } \\
\text { (measured) }\end{array}$ & $\begin{array}{c}\text { No. of Figures } \\
\text { (calculated) }\end{array}$ \\
\hline 543 & 86 & 86 \\
594 & 79 & 79 \\
604 & 77 & 77 \\
633 & 74 & 74 \\
\hline
\end{tabular}

of the scattering pattern has basically two components: (1) fringe component with higher frequency which is dependent on the refracted and reflected rays that do not cross the fibre core; and (2) a lower frequency component that modulates the fringes caused by interference between the refracted ray that traverses the core and the one that goes through the 
cladding only. This component can potentially be used to evaluate the fibre core parameters (e.g., refractive index and diameter). In general, the unique signature produced by such scattering is what makes the use of structured fibres as optical encoding keys promising [23]. Unfortunately, this complexity makes monitoring of the scattered signature from the side to monitor alignment and rotation for other applications unnecessarily challenging and complicated since phase information becomes critical. It is possible by taking this information and feeding it to a wavefront encoder (e.g., an array of liquid crystals or micromechanical (MEMs) mirrors) to manipulate the phase front of the incident light, the intensity at the center of the core can be actively maximized. Such a feedback system has allowed a random Ag based paint deposited onto a glass slide to focus light [32]. A simpler approach may be to perform alignment and rotation by monitoring a process within the core of the optical fibre.

\subsection{Measuring the Light Reaching the Core of the Structured Optical Fibre}

The object when inscribing gratings is to illuminate the core of the fiber with sufficient power density to induce the required refractive index change within the core glass in spite of this scatter. A preliminary analysis of the likely impact of scattering can be obtained through computer simulation. To date, an idealized 4-ring structure such as shown in Figure 1(a) has been simulated using ray trace software [26] and with finite difference time domain (FDTD) software [28]. A previous model [27] which examined grating inscription with multiple IR photons required a very tight $4 \mu \mathrm{m}$ focus of the light cone directly into the fibre core to generate the power density necessary to induce refractive index change in the core glass. Unfortunately, this is not representative of existing writing conditions employed in phase mask writing, where a plane wave illumination of the core from, for example, a frequency doubled $\mathrm{Ar}^{+}$(CW $244 \mathrm{~nm}$ ) laser or from an exciplex laser such as $\operatorname{ArF}(193 \mathrm{~nm})$.

Figure 4 shows the FDTD simulation of $193 \mathrm{~nm}$ TEpolarized light striking a fibre identical to that of Figure 1(a) from the side at two particular orientations [28]. The orientation at $0^{\circ}$ has more uniform distribution of UV light within the core region; however, a close inspection of the peak intensities reveals that whilst much less uniform, the orientation at $30^{\circ}$ has a very high-peak intensity at the back of the core, suggesting that there is additional lensing effects arising from the lattice itself, despite a greater amount of light scattered at this orientation. This is consistent with constructive and destructive interference of coherent light scattered by the lattice (our laser coherence is $>600 \mu \mathrm{m}$ ), as alluded to in previous sections. A conjecture would be that for high-exponent processes the $30^{\circ}$ orientation is preferred. On the other hand, for single-photon processes, such as those involved when a germanosilicate core is present, $0^{\circ}$ is preferred.

More recently, FDTD simulation was extended to a more complex 12-ring photonic crystal fibre, shown in Figure 1(b), with a highly photosensitive and very high-NA

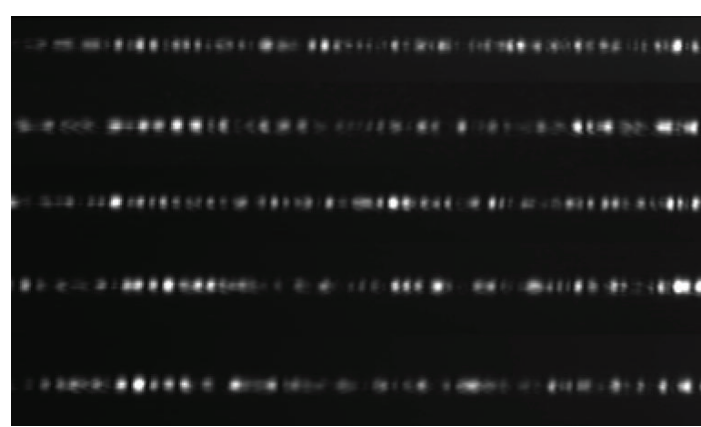

(a)

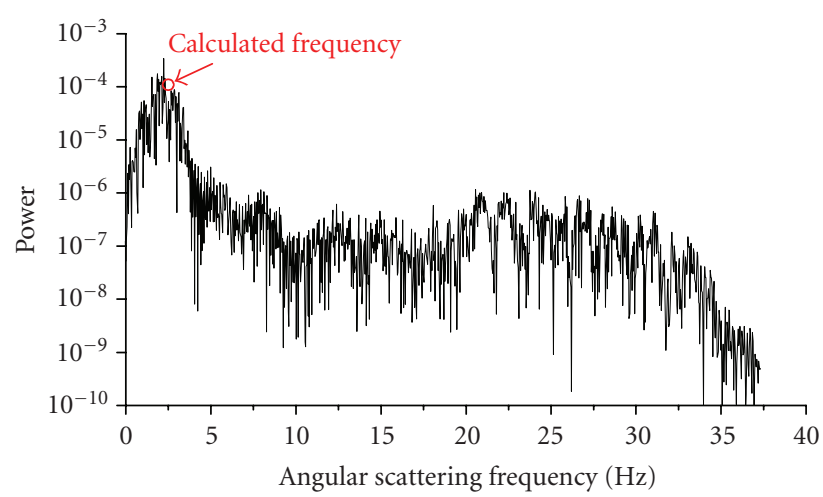

(b)

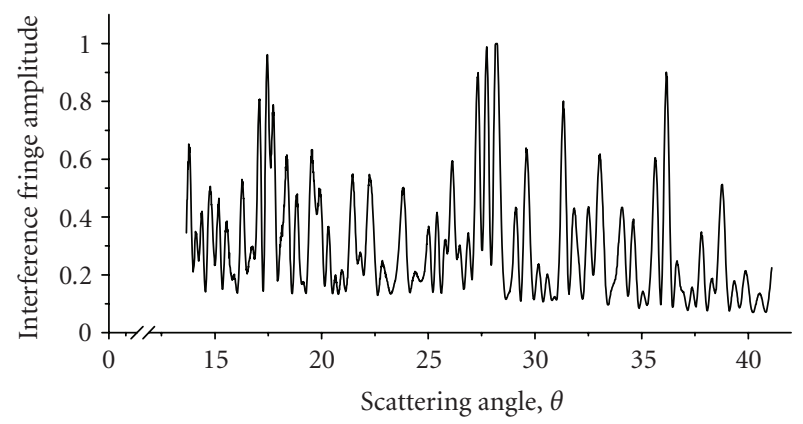

(c)

FIGURE 3: (a) Interference pattern images, (b) FFT of the scattered light at $543 \mathrm{~nm}$, and (c) intensity plot at $543 \mathrm{~nm} \mathrm{[23].}$

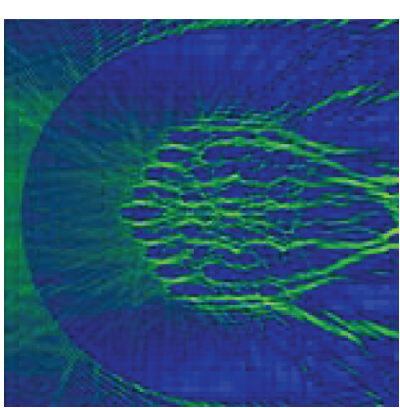

(a)

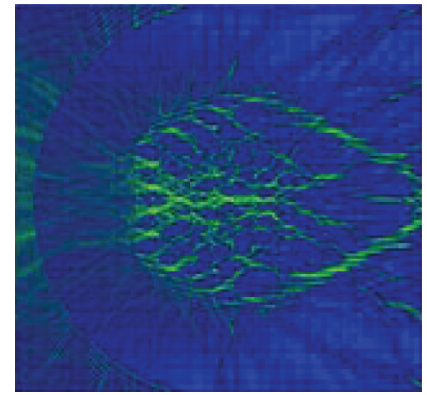

(b)
FIGURE 4: $193 \mathrm{~nm}$ light (TE) focussed and scattered within a 4-ring photonic crystal fibre (Figure 1(a)): (a) $0^{\circ}$ (ГM) and (b) $30^{\circ}$ (ГK) orientations [28]. 


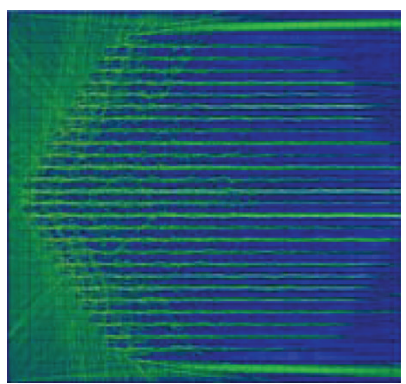

(a)

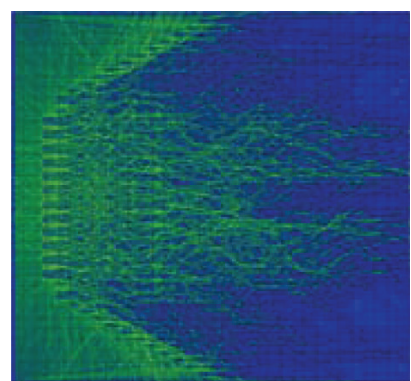

(b)
FIGURE 5: $193 \mathrm{~nm}$ light (TE) focussed and scattered within a 12 ring photonic crystal fibre (Figure $1(\mathrm{~b})$ ): (a) $0^{\circ}$ (ГМ) and (b) $30^{\circ}$ (ГK) orientations [30].

core designed for nonlinear work. The results are shown in Figure 5. Given restrictions on computational time, the full fibre structure, including the outer fibre diameter, was not simulated. A plane wavefront is used to excite the entire structure, approximately simulating the front expected within the Rayleigh range of a focusing lens. The effect of not considering the outer fibre diameter, and therefore wavefront curvature, results in the $0^{\circ}$ orientation light seemingly zipping through the "wave guiding" regions between the holes. For this particular fibre this is especially important because the core is a triangular region defined by four defects (or four missing holes) in the center. Further, the central region of this core is composed of germanosilicate for added photosensitivity. However, it would appear based on this simulation that the transmitted light, whilst significant, actually bypasses the photosensitive region in the center. One could assume, therefore, that the preferred angle is $30^{\circ}$. Evidently, the expected results are highly sensitive not only to the lattice parameters, but also to the core structure and outer fibre diameter as well-different fibres may very well have unique solutions and, further, regular structured fibres are likely to be distinct to those with quasiperiodic structures including, for example, Fresnel fibres with chirped Bragg structures.

To investigate the particular case of the 12-ring fibre described above, grating writing into the fibre was carried out using motorized controlled rotation [30]. The particularly high concentration of germanium in the photosensitive region means this fibre permits ready formation of type IIa gratings $[33,34]$. Therefore, information can be extracted in three ways: (1) average index shift from the Bragg wavelength shift, (2) index modulation from the grating strength, and (3) qualitative intensity variations based on the rollover from type I to type II a, which is known to be sensitive to writing intensity [35]. In order to calibrate the orientation, $244 \mathrm{~nm}$ light was used to directly excite germanium oxygen deficiency centers in the photosensitive core and blue luminescence monitored as a function of rotation angle. Although grating writing is carried out at $193 \mathrm{~nm}$, the amount of blue luminescence generated by this wavelength is considerably less than that generated with $244 \mathrm{~nm}$. Figure 6 shows an image of the end face of the

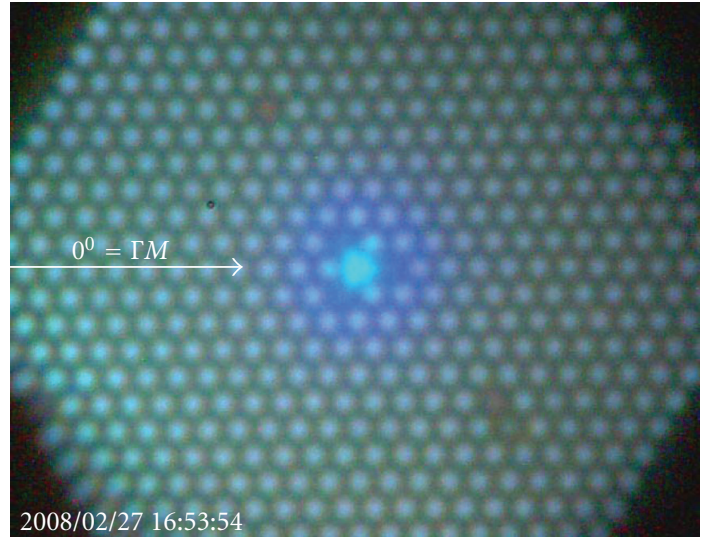

(a)

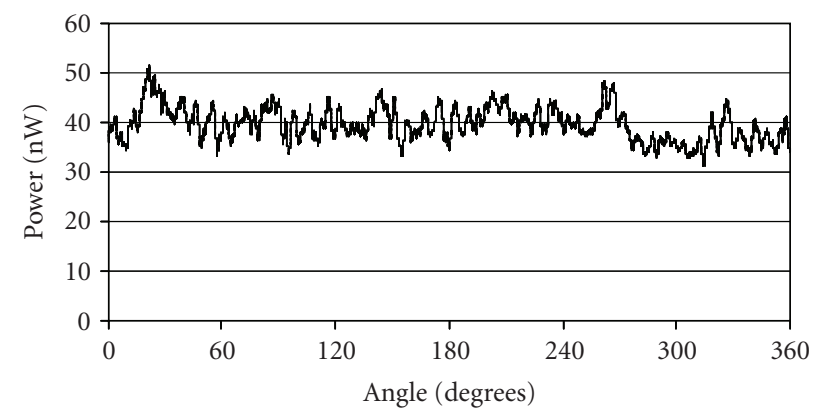

(b)

Figure 6: Blue luminescence propagating along the core of the 12 -ring photonic crystal fibre is readily imaged. Also visible is the luminescence supported in the holes akin to whispering gallery modes of a hollow waveguide. The profile of the blue luminescence as a function of rotation angle is shown below [28-31].

fibre with the photosensitive region brightly illuminated and the monitored profile as a function of rotation. Based on these results, the optimum angle of excitation is found to be $21.5^{\circ}$. However, luminescence is reasonably strong elsewhere including for $0^{\circ}$ and $30^{\circ}$, where it is identical. These two angles are important as these orientations are easily aligned visually-the sensitivity of $21.5^{\circ}$ demands greater care in alignment. The observed variation in signal reaching the photosensitive core, including experimental error, is approaching $30 \%$ which is significant.

The experimental grating results at $0^{\circ}, 21.5^{\circ}$, and at $30^{\circ}$ are shown in Figure 7. As expected from the luminescence studies, the average index growth profiles, from positive to negative index change for a series of gratings are found to overlap exactly for $0^{\circ}$ and $30^{\circ}$. The actual grating strengths varied in part because of the interference of the two modes of this fibre over the short length spliced between two standard single mode telecommunications fibres. It is observed that for all cases, the rollover from type I to type IIa (Type In) grating occurs at the same cumulative fluence despite the fringe contrast variations also indicating that the same intensity is present for the two angles.

The results at $21.5^{\circ}$ were found to have a larger average index, consistent with the luminescence studies-however, 


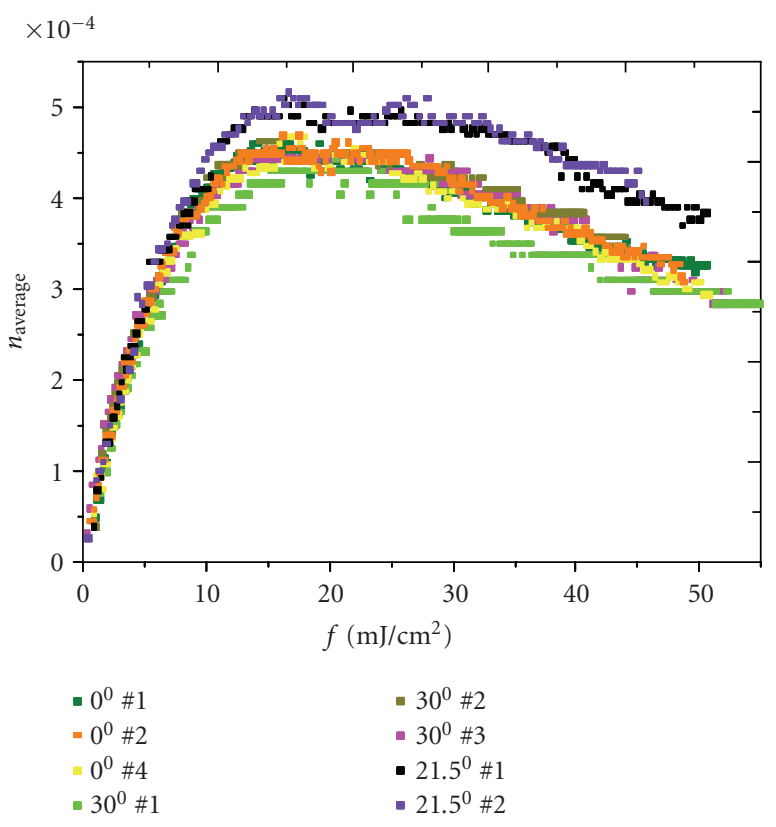

(a)

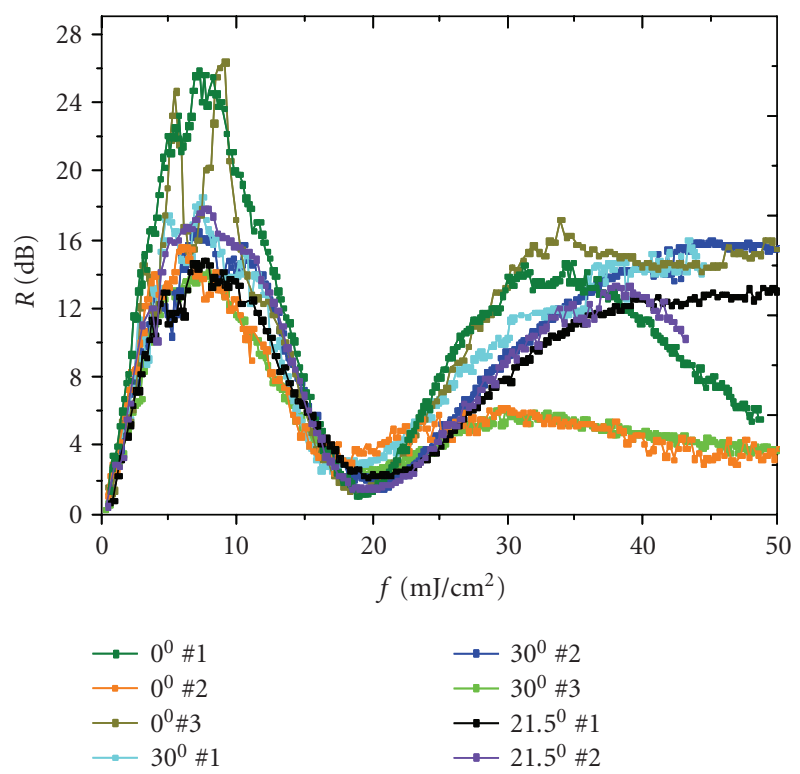

(b)

Figure 7: Type I and Type IIa evolution in a 12-ring photonic crystal fibre with germanosilicate core. (a) Average index (extracted from $\Delta \lambda$ shift) and (b) grating strength as a function of fluence [34].

the experimental variation over a series of runs at this angle was much more significant so the results in themselves were not conclusive at this stage. More interestingly, we observed that the asymmetric frame of reference of the structure with respect to the irradiating beam meant considerable asymmetry was introduced into the core and the grating had large birefringence $\left(\sim 1 \times 10^{-4}\right)$ as shown in Figure 8 [34].

In both simulations described above, only the orthogonal incidence of a plane wave to the fibre has been considered. Whilst this has been sufficient for good qualitative agreement

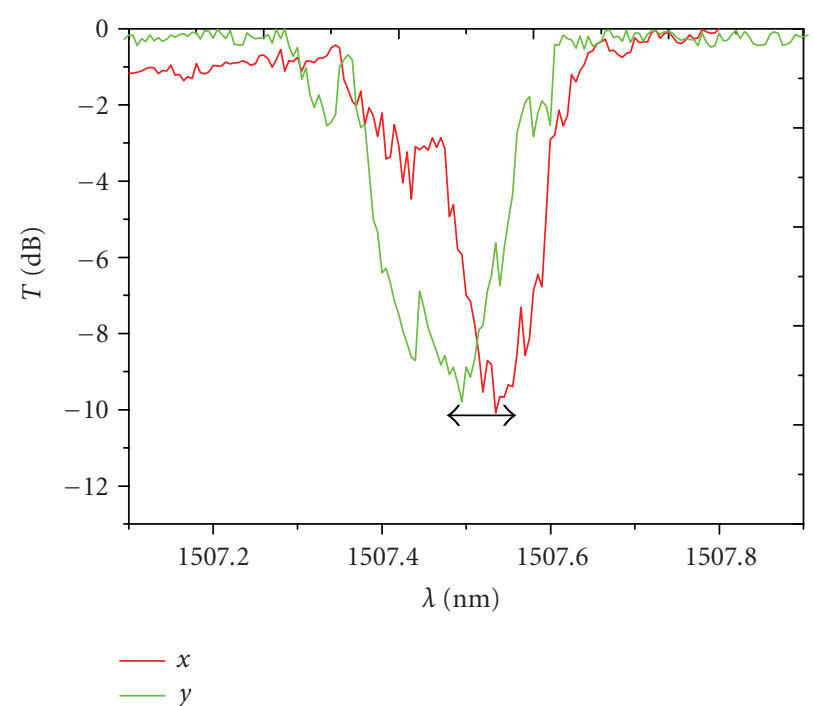

FIGURE 8: Grating induced into fibre core at $21.5^{\circ}$ incident writing beam to the $0^{\circ}$ axis. Large birefringence is observed $\left(\sim 1 \times 10^{-4}\right)$.

with experiments, in practice, the diffraction orders of a phase mask setup, or the angles of a two beam interference setup, have a vectorial component typically $\sim 30-35^{\circ}$ to the fibre axis for grating writing in the telecommunications window. The main impact of this, however, will be to affect the quality of the fringe pattern at the core rather than the total cumulative fluence provided care is taken with overall alignment. Any deviations, such as fibre tilt, will complicate results.

In summary, the general problem of scattering demands that attention is paid to the rotationally variant scattering properties of most structured optical fibres, including crystalline (photonic crystal) and quasicrystalline (Fresnel) and random structured fibres. The most obvious solution described above is rotational control of the fibre positioning during grating writing. Practical alignment of fibres can be achieved in several ways including monitoring of defect luminescence [28-30] or, for example, in other fibres such as $\mathrm{Er}^{3+}$-doped fibres, rare earth emission during UV excitation $[28,29]$ with rotation. Alternatively, the more sophisticated and active approach using measurement of the transmitted wavefront across the fibre to feedback into wavefront phase adjuster as mentioned earlier can be adopted. This would depend on the availability of suitable phase adjusters such as liquid crystal arrays [32] or MEMs mirrors and may not be feasible for shorter UV wavelengths, but amenable to longer wavelengths including those used in multiphoton grating writing.

\section{Reducing Scattering}

We have discussed both type I and type IIa formation in photonic crystal fibres containing germanosilicate and in all respects the photosensitive properties are well established in conventional fibre grating writing, although the cumulative fluencies tend to be much higher as a result of the scattered 
light away from the core. However, the structured optical fibre has demonstrated how an unconventional design can be used to obtain further spatial information to help improve the understanding of the physical origins associated with such gratings generally. Previous work using spatial characterization to help elucidate underlying mechanisms used the differences seen by a fundamental and a higherorder mode in a two-mode conventional optical fibre [36]. The results in Section 2 are consistent with that work.

It is clearly important to control rotational variance both in terms of reproducibility and in terms of grating writing efficiency. This is especially so for higher-exponent processes that require substantially more photons per unit area. An alternative approach to deal with this problem is to consider inserting material into the holes, that is, index matched to the silica so that the scattering is reduced or removed altogether. Whilst this is certainly a challenge for $193 \mathrm{~nm}$ grating writing, since there are few materials that transmit and do not ablate at such short wavelengths, this approach has been successfully used to improve grating writing at $248 \mathrm{~nm}[8]$.

A similar approach permitted the inscription of femtosecond-written damage gratings into structured optical fibre-by filling an air-clad fibre with a $\mathrm{Yb}^{3+}$ doped germano-aluminosilicate step-index core (shown in Figure 1(d)) with index matching gel, a near IR $(800 \mathrm{~nm})$ femtosecond laser was able to access the core of the fibre and inscribe a high-temperature resistant damage grating [20]. A micrograph of two filaments induced on one side of the air-clad core is shown in Figure 9. Prior to this, the only gratings written directly into large diameter air-clad structured fibres, designed for high-power laser operation, were done so by using single photon excitation with either $244 \mathrm{~nm}$ or $193 \mathrm{~nm}$ [19]. This result was the first reported result of a grating written directly into the active medium of any large diameter fibre designed for high-power laser operation.

From here on we review the increasing interest in higherexponent grating writing, including two-photon absorption with UV lasers and multiphoton absorption using femtosecond laser systems.

\section{Hydrogen Loading}

Another approach to reduce the impact of scattering in single-photon grating writing is to use hydrogen loading to maximize the photosensitivity available. However, the existence of the air holes surrounding the core poses a challenge for hydrogenation as it easily outdiffuses through the holes and escapes from the fibre by the end facets, which drastically reduces the photosensitivity. A practical solution is to seal the fibre end facets before hydrogen loading [37]. For the 12-ring photonic crystal fibre shown in Figure 1(b), this was done by fusion splicing the PCF length with lengths of standard SMF28 fibres on both sides. A number of problems are raised by splicing such different fibres, including (a) mismatch in fibre $\mathrm{V}$ parameter, (b) the existence of higher-order modes and intermodal interference fringes [38], and (c) splice integrity whilst minimizing losses.

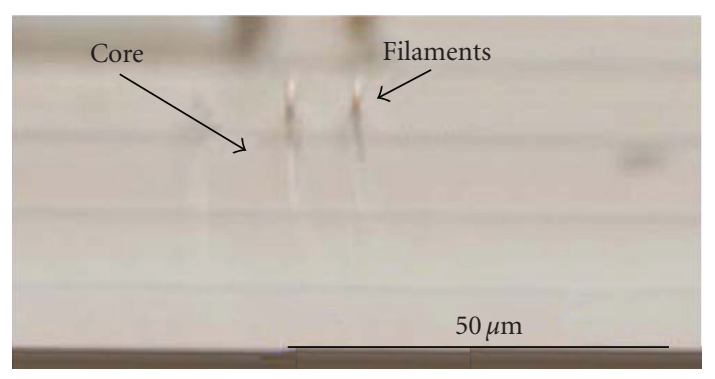

Figure 9: Femtosecond-induced filaments observed by optical microscope through the air cladding structure filled with index matching gel [20].

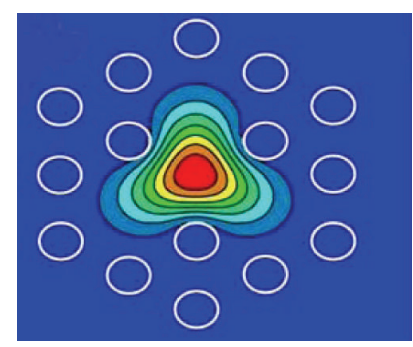

(a)

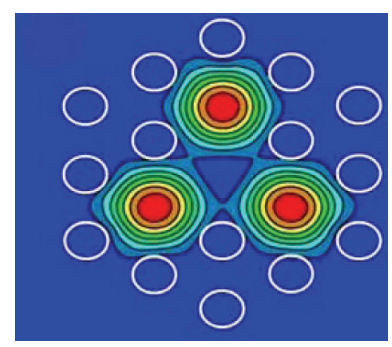

(b)
Figure 10: Calculated mode field profiles of the (a) fundamental mode and (b) the higher-order mode, which is made up of three nondegenerate but similar components, supported by the 12-ring photonic crystal fibre in Figure 1(b) [33, 34].

For the 12-ring fibre, insertion losses below $2 \mathrm{~dB}$ were obtained by monitoring coupling to the mode from the SMF28 to the fundamental mode of the structured fibre. This particular fibre has an effective higher-order mode that rests within the three lobes of the triangular core, as shown in Figure 10 and great care is required to avoid coupling into it. After splicing the samples, typical hydrogen loading conditions are $180 \mathrm{~atm}$ at $80^{\circ} \mathrm{C}$ for 5 to 7 days to ensure the holes are saturated in pressure and equilibrated to the pressure within the glass. Within this particular fibre, gratings $>30 \mathrm{~dB}$ in strength were possible [8]. In all respects, the gratings are again identical to standard gratings written into conventional hydrogen-loaded germanosilicate fibres. Similar thermal annealing is observed (Figure 11) [39]. The estimated effective thermooptic coefficient is $12.3 \mathrm{pm} /{ }^{\circ} \mathrm{C}$, which agrees well with values obtained on gratings written in high-concentration Ge-doped, conventional step-index silica fibre [40].

Hydrogen loading was also used to demonstrate the first femtosecond gratings in pure silica fibres using $266 \mathrm{~nm}$ femtosecond light [10]. In these experiments, the grating decay indicated they were relatively unstable and probably related to direct single-photon $266 \mathrm{~nm}$ hydride formation well below the damage threshold of the glass. Scattering, along with absorption by hydrogen species, prevented higher-exponent excitation below the band edge. Generally, below the damage threshold it has been shown that there is largely no difference between gratings written with UV femtosecond $244 \mathrm{~nm}$ 


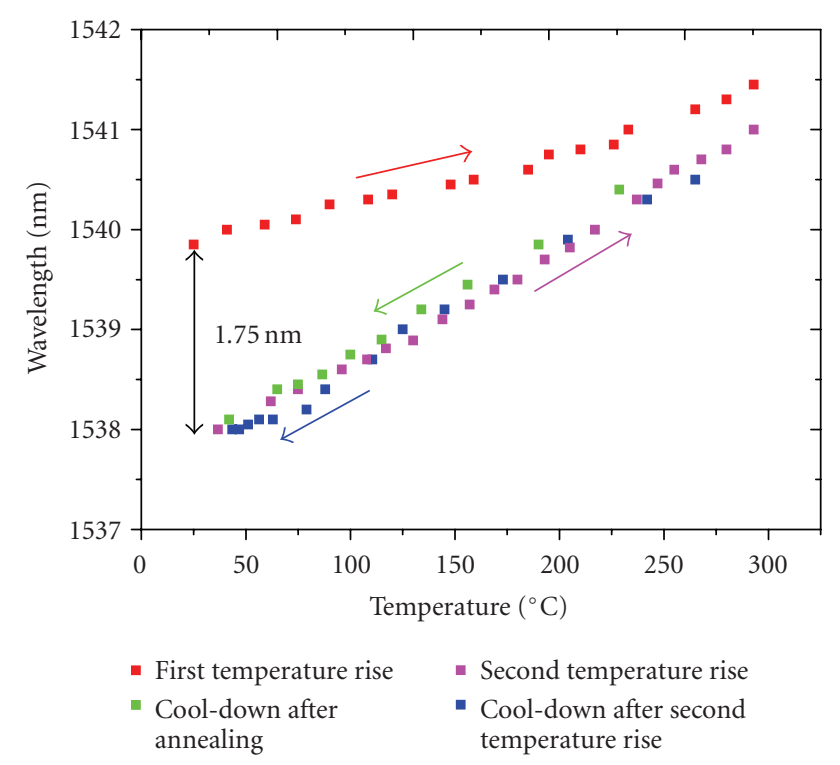

Figure 11: Temperature cycling of hydrogen-loaded 12-ring photonic crystal fibre grating (Figure 1(b)). The initial drop in Bragg wavelength arises from outdiffusion of the hydrogen, which raises the effective mode index by nearly $2 \times 10^{-3}$. Beyond this, the index change is stable after repeated cycling from room temperature to $300^{\circ} \mathrm{C}$. There is no observed hysteresis [39].

lasers and gratings written with CW $244 \mathrm{~nm}$ [41]. Thus no clear advantage has been established for the use of a femtosecond laser for writing of type I gratings.

\section{Two Photon Grating Writing}

Two photon absorption of UV light permits access below the band edge of glass to excite $\mathrm{Si}-\mathrm{O}$ bonds, or other component in other glasses, that make up the glass network. Unlike single-photon excitation, defects are largely circumvented. Rather, softening and/or breaking of the oxide bonds of the network allow glass structural rearrangement, and therefore densification, to take place. The energy required for this process need not exceed the damage threshold, thereby avoiding cascaded ionization typical of femtosecond laser irradiation in addition to fracturing within the network associated with longer pulse excitation (type II gratings). Cumulative fluencies, therefore, tend to be large in comparison with other grating writing methods, particularly if the energy density is sufficient to soften the bonds and allows thermal relaxation into a different density glass, akin to thermal quenching of glass in a controlled fashion [42]. Consequently, this densification grating is usually of lower loss than damage gratings although within structured optical fibres this will give rise to surface corrugations within the air holes [43]. Generally speaking, the process becomes more efficient the deeper into the UV the excitation occurs and therefore $193 \mathrm{~nm}$ from the excited state complex (exciplex) of an ArF laser is significantly more efficient than, for example, $248 \mathrm{~nm}$ from a $\mathrm{KrF}$ laser. From the perspective of inscribing a periodic structure on dimensions approaching

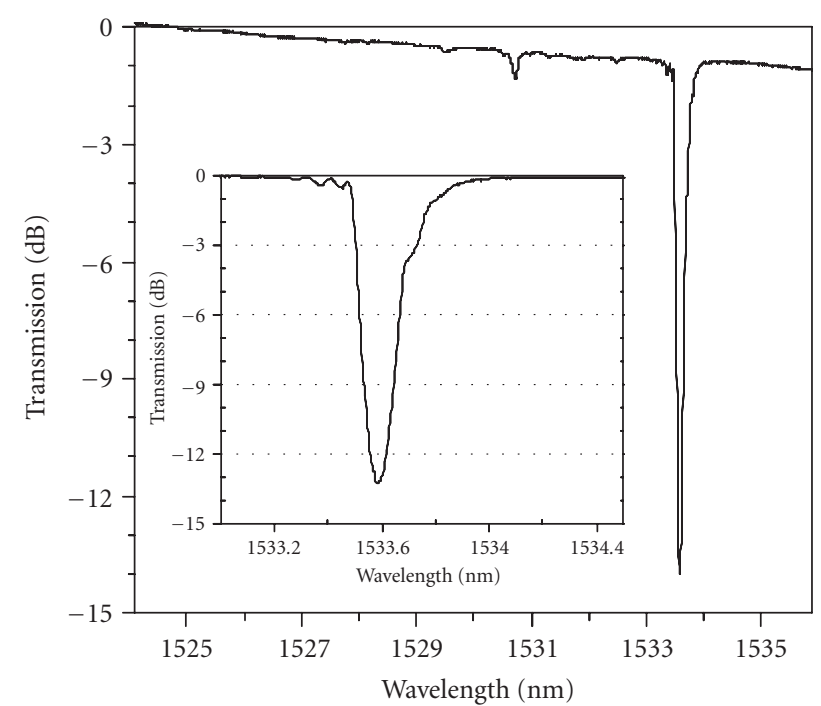

FIGURE 12: Grating in pure silica single-material photonic crystal fibre [14].

the nanoregime, this also leads to improved localization and fringe contrast. Going to shorter wavelengths, such as $157 \mathrm{~nm}$ generated from the excited state dimer (excimer) of an $\mathrm{F}_{2}$ laser, poses practical challenges in terms of delivery optics and the need for a vacuum.

\subsection{Densification Gratings in Single Material-Structured Fibres}

Figure 12 shows the first transmission profile of a densification grating written into a 4-ring, single-material, silicaphotonic crystal fibre similar to that in Figure 1(a) [14]. In such a fibre, the densification leads to corrugations within the air channels of the structured fibre, which was confirmed in studies involving the flow of ice formation inside water filled holes of such a grating [43]. Figure 13 shows the reflection spectrum of a similar grating written into the Fresnel fibre shown in Figure 1(c) [15]. The optical mode peaks in the center of the central hole. Nevertheless, sufficient light extends into the ring of silica around the hole to "see" a reasonably strong grating, a good example of how a bandgap fibre is modified to have a hybrid structure with air and glass. Of course, in all-solid bandgap structures this is unnecessary but the applications become somewhat restricted. This method of grating writing is based on well-established densification of silica glasses under UV irradiation which precedes germanosilicate grating writing by decades [44-47]. More recently, femtosecond lasers under strict control can operate just below the glass damage threshold to achieve similar results [48].

\subsection{Densification Gratings in Nongermanosilicate Core Structured Fibres}

Whilst two-photon absorption of UV light may require long cumulative fluencies for pure silica [14], when the glass 


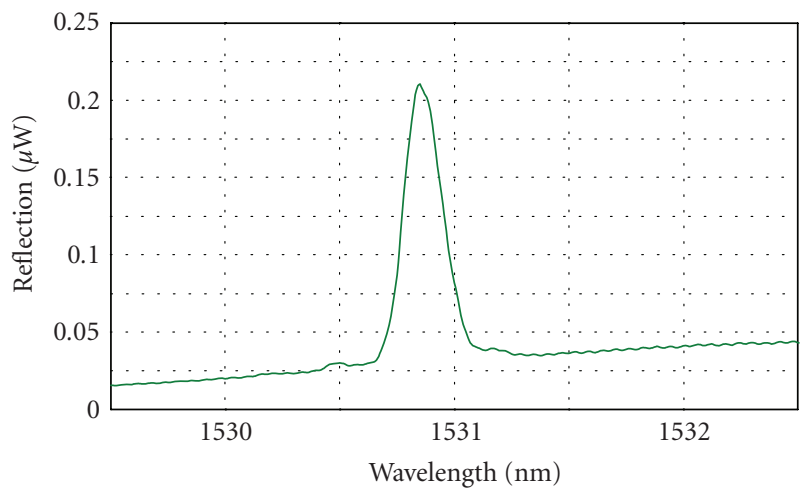

FIGURE 13: Reflection spectra of a $5 \mathrm{~dB}$ strong grating within the Fresnel fibre shown in Figure 12 [15]. The optical intensity of a propagating mode peaks in the core hole.

composition is mixed, the photosoftening threshold can be reduced and the writing processes enhanced and accelerated significantly. In the case of nongermanosilicate step-index core-structured fibres, competitive writing times with conventional grating writing have been demonstrated for the fabrication of $\mathrm{Er}^{3+}$-doped aluminosilicate photonic crystal fibre distributed Bragg reflector (DBR) and distributed feedback (DFB) fibre lasers [16-18]. By way of example, the characteristic output of a DFB photonic crystal fibre laser fabricated in this fashion is shown in Figure 14. Writing times are now $<20$ minutes in such fibres depending on the device parameters sought. Given that much of this change occurs within the step-index region of the structured fibre core, corrugations are not so prominent. It is important to verify that the writing excitation process is two-photon even if the subsequent glass relaxation is unlikely to be directly correlated with the excitation.

Unfortunately, the small window for efficient twophoton induced index change just below the damage threshold, along with the error window found when dealing with scattering and other issues, has prevented to date a direct measure of the quadratic dependence with intensity of index change. Instead, as mentioned above, one approach is to infer the presence of densification itself from restrictions in ice flow that give rise to distortions in the grating profile and eventually fibre cracking [43]. This takes advantage of the fact that water freezing within small capillaries leads to a super-cooled liquid phase between the ice and the glass interface which "lubricates" flow of the ice as it expands from the liquid state. This super-cooled layer is small, and therefore the lubrication process is impeded in the presence of corrugations. However, for this fibre the changes occur within the step-index portion and may not translate to the surrounding silica walls of the air holes. A less direct approach is to repeat the experiments in a preform slice from which the doped core inserted into the structured optical fibre during fabrication is made $[49,50]$. Direct depressions arising from densification can, therefore, be measured using atomic force microscopy (AFM), laser diffraction from periodic densified regions, and phase microscopy. Figure 15 shows the measured dependencies determined from such

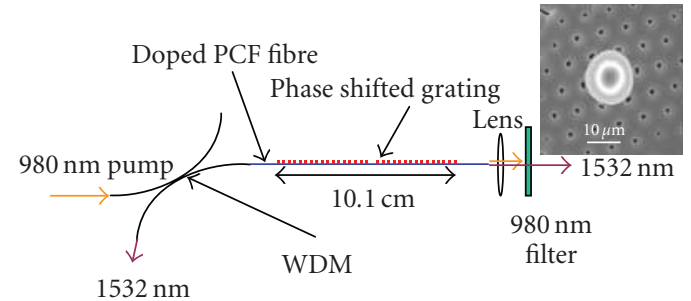

(a)

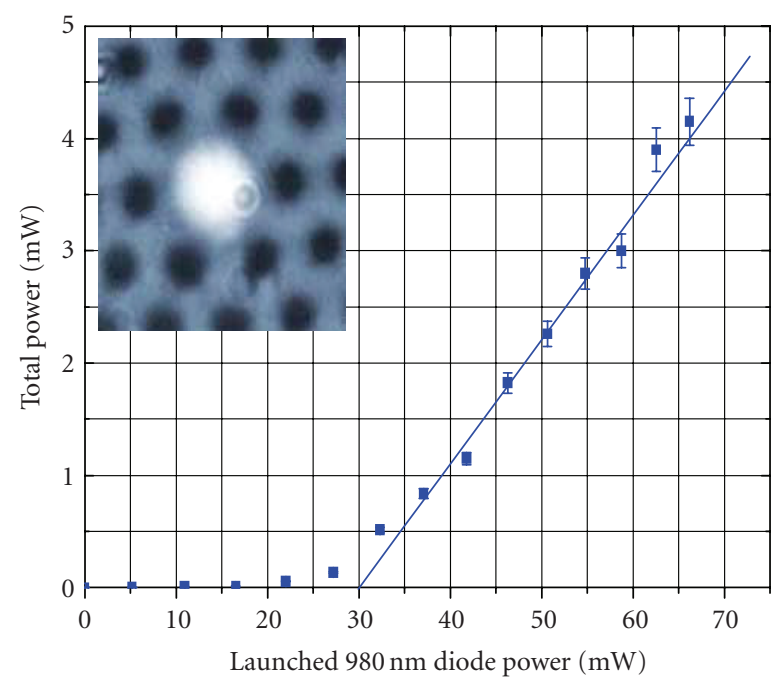

(b)

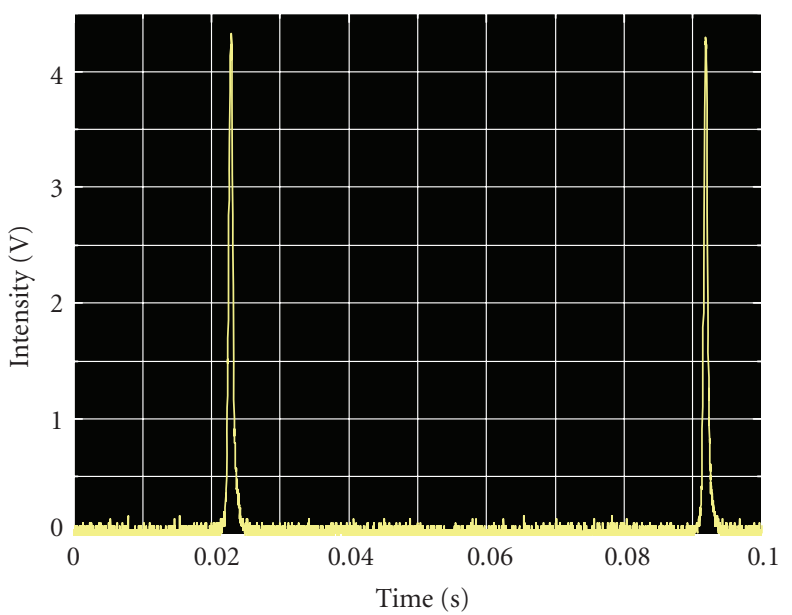

(c)

FIGURE 14: Characteristics of a DFB photonic crystal fire laser. The grating is written by a 2-photon process which avoids the need for hydrogen loading. The top shows a schematic of the setup and a profile of the measured laser output. Center is the characteristic power curve-inset shows the doped section illuminated with white light. Bottom shows the laser operates single polarization. The measured linewidth by self-heterodyne is $<50 \mathrm{kHz}$ [17].

techniques for both $193 \mathrm{~nm}$ single-photon excitation of a germanosilicate perform and what is thought to be a twophoton excitation of an $\mathrm{Er}^{3+}$-doped aluminosilicate perform 


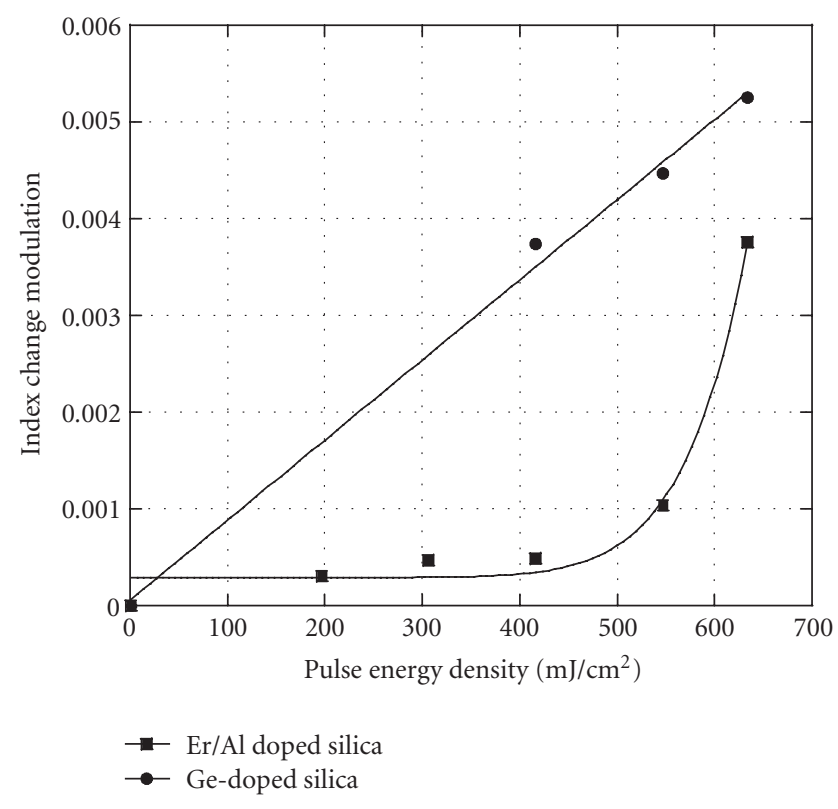

Figure 15: Index modulation as a function of pulse energy for a germanosilicate preform (linear) and an $\mathrm{Er}^{3+}$-doped aluminosilicate preform (non-linear). The latter preform is a slice from that used to draw the fibre into which the DFB of Figure 12 was drawn $[46,47]$.

[46, 47]. Densification has been confirmed but further work is necessary to clarify the complex nonlinear behavior observed.

\section{Femtosecond Laser Written Gratings}

Multiphoton absorption with exponents $>5$ using ultrashort pulses ( 100-600 femtoseconds typically) is an increasingly popular way of writing long period and Bragg gratings either into the ODC absorption band (type I gratings) or into the glass band edge directly (femtosecond type II gratings) into both conventional [51-58] and structured optical fibres $[20,21]$. Although using long pulses with exciplex lasers can access multiphoton processes [59], the much shorter timescale of femtosecond excitation means that electronic avalanche ionization can be generated locally on a timescale much faster than any direct photon decoupling away from the excitation site. The large rapid local index change leads to filamentation in part arising from self-focusing effects by the generated plasma, which acts to prevent the collateral damage more familiar to long pulse lasers. Given that the spatial extent of these cylindrical filaments is commensurate or smaller than the probing wavelength within the VIS-NIR window, significant Mie scattering can be generated if not adjusted. These gratings, therefore, have significant diffractive scattering losses increasing toward shorter wavelengths $[60,61]$. Despite these large scattering losses, these gratings have been used to successfully demonstrate a stable $100 \mathrm{~W}$ $\mathrm{CW} \mathrm{Yb}^{3+}$-doped fibre laser operating $\sim 1 \mu \mathrm{m}[62]$. Since the pump wavelength is coupled into the inner cladding, the grating losses at the pump wavelength are minimized. This is a tremendous improvement over the failure of type I gratings to perform within a similar fibre laser operating at only $15 \mathrm{~W}[19,63]$. However, the extremely serious problem of very high-optical fields giving rise to large local temperatures within $\mathrm{Yb}^{3+}$-doped optical fibres, a work horse of high-power fibre lasers up to the $\mathrm{kW}$ regime, has not been fully resolved. Femtosecond gratings are now known to self-erase within such lasers when operating as a Q-switched laser [64], a phenomenon of considerable scientific and practical interest. At very high powers, therefore, at this point in time it remains preferable to keep the gratings external to the active medium. This stringent limitation is almost certainly most serious for $\mathrm{Yb}^{3+}$ which is known to have a high-thermal nonradiative component as well as unusual resonant excitation processes which may couple light directly into the band edge and consequently also leading to index change [65].

To summarize the processes briefly, it has been well established that the damage mechanism can be described in terms of multiphoton ionization of electrons, heating of free electrons, and a shock-like transfer of the energy to the lattice. This process is well known as ablation of a surface material-however, when localized within a material by these multiphoton processes, the shockwave leads to what has been called a microexplosion [66]. At sufficiently high intensity, this allows direct void formation to also be induced in the glass, an approach that appears to be aided by having a photosensitive dopant within the glass. One example is cerium-doped glass that has been used to make 3-D microfluidic channels [67]. Associated with this structural change is densification when the intensity is low [68]. The ionization mechanism underpinning femtosecond laser operation is therefore not dependent exclusively on the wavelength. Both $266 \mathrm{~nm}$ via two photons [69] and $522 \mathrm{~nm}$ via few photons have been used to achieve similar results [70].

\subsection{Gratings in Single-Material Photonic Crystal Fibres}

Femtosecond grating writing in single-material all-silica photonic crystal fibres has recently been reported for few ring structures [21]. The authors comment that scattering issues are the primary reason why this has not been extended beyond a few rings. Tapering of the fibres is one solution to help reduce this scattering, since the period of the hole spacing is now smaller than the wavelength, reducing scattering substantially.

\subsection{Gratings in Air-Clad Structured Fibres}

Earlier, we described grating writing through an air-clad fibre by using index matching gel to remove the Fresnel reflections from the hole air-silica interface. In this way, femtosecond gratings were inscribed into air-clad structured fibres, where only a single ring of air holes defined the inner cladding of a double-clad fibre with a central step-index core containing $\mathrm{Yb}^{3+}$ ions for high-power fibre lasers [20]. Figure 1(d) shows the cross-section of such a fibre. This fibre was used using 


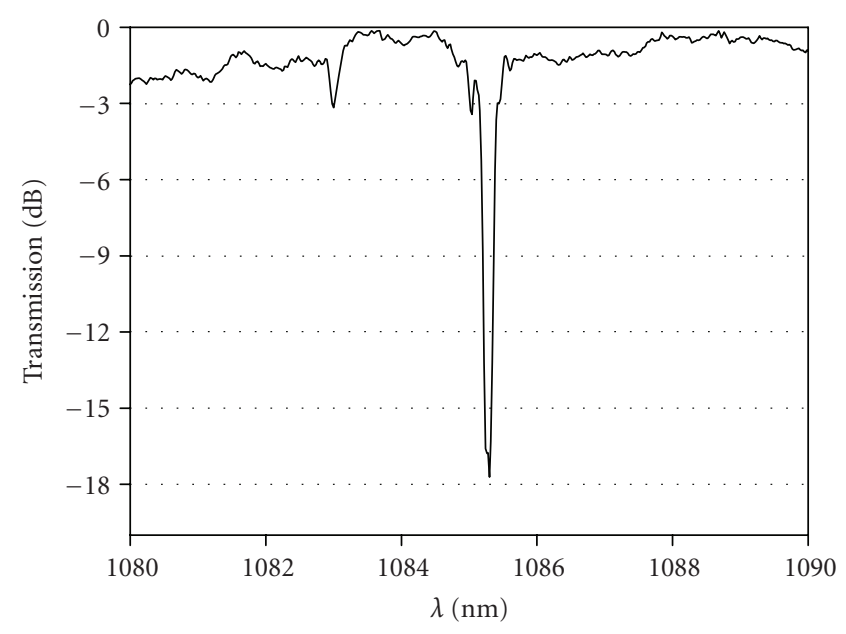

FIGURE 16: Transmission spectra of a femtosecond grating written into the $\mathrm{Yb}^{3+}$-doped core of an air clad fibre $400 \mu \mathrm{m}$ diameter [20]. A higher-order mode grating is also observed.

bulk mirrors and end face reflection to generate $>100 \mathrm{~W}$ fibre laser power.

Bragg gratings were produced using a femtosecond laser $(800 \mathrm{~nm}, 100$ femtoseconds, $1 \mathrm{kHz})$ period by period as the beam is scanned over the fibre. A transmission spectrum with $16 \mathrm{~dB}$ rejection is shown in Figure 16. Similar laser performances to the $100 \mathrm{~W}$ fibre laser reported much later using femtosecond gratings in conventional double-clad fibres [62] can be expected. The use of a gel made it difficult to remove after grating writing to prevent highpower fibre laser operation. This could be improved using index-matched liquids rather than gel so that heating can be used to subsequently remove the liquid. Characteristic of such gratings is the observation of substantial diffractive scattering at short wavelengths shown in Figure 17 [20]. Such losses reduce the overall efficiency of high-power fibre lasers since the pump wavelength in particular experiences loss. A detailed and systematic study of this loss confirmed similar properties to conventional type II gratings $[60,61]$ despite the much more uniform nature of the processed regions. Although the structural nature arising from photoinduced ionization and melting may differ as a result of different excitation and local quenching rates, this is unsurprising given both are damage gratings written above the damage threshold of the glass. In fact, the uniformity leads to efficient side diffraction light travelling within such a fibre, which can be utilized to make an ideal component for a compact fibre spectrometer, suitable for a number of applications including sensing, monitoring, and external coupling of fibre laser light.

\section{Properties and Applications}

A key factor that separates structured fibre gratings from conventional gratings is the composite nature of the fibre. By controlling the hole distribution and size, mechanical properties are altered, including acoustic and pressure

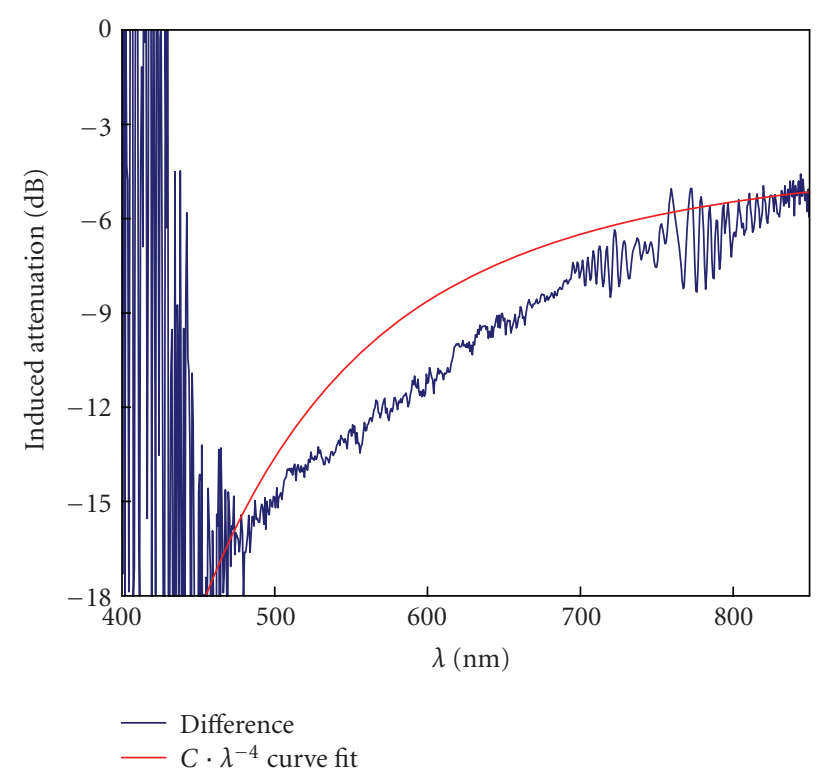

FIGURE 17: Induced short-wavelength loss of air-clad grating shown in Figure 14. An almost linear dependence is observed consistent with Mie scattering [20].

response, as well as even more basic properties such as strain optic coefficient. Inserting material gives greater scope for adjusting composite system properties such as the effective thermooptic coefficient discussed earlier. The flexibility in adjusting such parameters also offers new solutions to perennial problems including sensors perspective, separating strain, and thermal properties. Some of these properties are reviewed here.

\subsection{Strain and Temperature Response}

An important distinction between conventional step-index fibres and structured optical fibres, even those with a stepindex core, is the striking differences in properties possible between the so-called fundamental mode and the higherorder modes. Depending on the design, losses and effective indices are comparable with a step-index fibre. In an ideal fibre, losses are almost entirely due to evanescent field penetration outside of the core (except in the special case, where the cladding may extend to infinity). This is not true for a structured optical fibre, where additional leakage loss between air holes or filled channels occurs. On the other hand, the high-index contrast between, for example, air and silica means the evanescent field peaks and is much more tightly localized to the interface, although losses can still be higher if the hole size is sufficiently small to allow coupling between one side and the other, perhaps through excitation of whispering gallery modes. These properties are formulated by the different effective fibre mode indices which are the index of the composite system. It may be expected that the properties of structured fibres will differ and vary to those of step-index fibres. Given that in the first instance the fundamental leaky mode of the structured fibre has a $k$ vector along the fibre axis and therefore the role of leakage 
loss is somewhat suppressed, the fundamental mode may not be so distinctive in behavior to that of the step-index analog. This forms the justification for the step-index approximation of simple-structured fibres, where the cladding index is, on average, lower than the core index. On the other hand, tightly confining such a fibre will deliver noticeable and application worthy differences. By adjusting the crystal lattice parameters of a photonic crystal fibre appropriately, unique composite system properties can be displayed. A good example is the demonstration of dual dispersion compensation and Raman amplification [71].

In contrast, higher-order modes will be significantly affected by leakage loss and greater evanescent field penetration into the holes. The extreme example of this is the air-clad fibre shown in Figure 1(d), where diffractive scattering plays a critical role in ensuring mode mixing and overlap with the core is high $[72,73]$, an important advantage that permits air-clad high-power fibre lasers to retain symmetry. In practice, even the higher-order modes of an ordinary regular photonic crystal fibre are far more sensitive to the cladding structure and enable practical sensing to be developed [74]. Even over short lengths supposedly, single-mode photonic crystal fibre can support higher-order leaky modes that lead to analogous intermodal interference observed with short lengths of standard single-mode telecommunications fibre [75]. It is noted within the literature that many gratings written into single-mode structured fibres have an observable higher-order mode resonance $[9,14,20]$.

It is this difference between the well-confined fundamental mode and the higher-order modes that allows a qualitative study of the properties of a structured optical fibre using Bragg gratings to determine both effective strain and thermooptic coefficients and compare with those obtained for standard optical fibres. In previous work, fiber Bragg gratings, written by two-photon excitation, within a twomode solid core photonic crystal fiber, which has a cladding region defined by a silica layer containing a triangular lattice of air-channels, were used to obtain the strain and temperature dependence of the structured fibre. The core is composed of an inner, doped core, and one outer silica ring - the presence of $\mathrm{Er}^{3+}$ plays no important role in the Bragg resonance dependence with strain [76]. The effective indices of the two modes give rise to two distinct grating peaks (Figure 1)—we note that this higher-order mode is made up of slightly nondegenerate components [18] but the resolution used was not able to separate these components. The modes are determined by the corresponding fractional powers $(\eta)$ of each mode within the higher-index-doped region (0.002) and in the surrounding silica. Leakage phenomena play an important part in determining and defining the mode field radius and therefore the fractional distribution of light of the modes either in the doped core or in the silica. Therefore, those modes with large transverse vector components will be sensitive to changes in the hole shape and stress between the holes in the structure arising from, for example, tensile or compressive stress applied along the fiber.

But it is also clear that this sensitivity will very much depend on air fraction, the regularity of the lattice, the constituent materials, and the type of index guidance. For example, the Fresnel fibre described earlier has much less air fraction, no regularity, is single-material all-silica, and the mode is largely determined by diffractive guidance even for the fundamental mode rather than an average step-indexlike effect. Therefore, a comparison of both temperature and strain effects of the fundamental mode within a singlematerial Fresnel fibre and a single-material photonic crystal fibre will further illustrate just how much flexibility in design exists by controlling structure alone.

\subsubsection{Temperature Dependence}

\section{(a) Fundamental versus Higher-Order Modes}

From Figure 18, the transmission spectrum of a 4-ring photonic crystal fiber with a fibre grating has two reflection bands corresponding with the two modes it is designed to support, one at longer wavelengths $(1535.2 \mathrm{~nm})$ for the fundamental mode, and the other $\sim 4 \mathrm{~nm}$ at shorter wavelengths for the higher-order mode. By monitoring these peaks, it is possible to determine the observed shifts experienced by each mode with temperature or applied strain. Figure 19 shows the obtained temperature dependencies for each mode-both grating transmission bands are found to have a similar linear variation with temperature. These results are in accordance with the material properties of the fiber, since the thermal expansion coefficient, $\alpha_{\mathrm{SiO}_{2}}$, of the fiber $\left(\alpha_{\mathrm{SiO} 2} \sim 0.55 \times 10^{-6}\right.$ for silica $)$ is an order of magnitude smaller than the thermooptic coefficient-for germanium-doped silica core fiber this is $\left(1 / n_{\text {eff }}\right)\left(\partial n_{\text {eff }} / \partial T\right)$ $\sim 8.6 \times 10^{-6}$, which serves as a reference value [77]. From the grating response as a function of temperature, the fundamental mode wavelength dependence is $\partial \lambda_{\mathrm{B} 1} / \partial T=$ $19.72 \mathrm{pm} /{ }^{\circ} \mathrm{C}$, and for $1535.2 \mathrm{~nm}$ of $\partial \lambda_{\mathrm{B} 2} / \partial \mathrm{T}=20.14 \mathrm{pm} /{ }^{\circ} \mathrm{C}$. These correspond to $\left(1 / n_{\mathrm{effl} 1}\right)\left(\partial n_{\mathrm{effl}} / \partial T\right)=12.9 \times 10^{-6{ }^{\circ}} \mathrm{C}^{-1}$ and $\left(1 / n_{\text {eff } 2}\right)\left(\partial n_{\text {eff } 2} / \partial T\right)=12.8 \times 10^{-6 \circ} \mathrm{C}^{-1}$ which are nearly double that of pure silica although similar to doped optical fibres. This larger value is due to the $\mathrm{Er}^{3+}$-doped aluminosilicate core and it is not surprising that the higherorder mode, which sees more silica, has a slightly lower value. Therefore, the composite system behavior is expected on the basis of constituent component materials.

An alternative approach to cancel out the temperature dependence by filling the channels with a liquid that has a large negative refractive thermooptic coefficient, such as many organic liquids [78], was mentioned earlier. The primary advantage of this is within sensor applications the contribution to the observed grating shift from temperature is removed altogether leaving only the strain contribution to be measured. This is important for many strain and pressure sensors. It is also a very simple means of packaging a grating - a viscous fluid within the channels can also serve to dampen external noise parameters.

\section{(b) Fresnel versus Photonic Crystal Fibre}

Figure 20 shows the relative wavelength shift of a Bragg grating written by 2 -photon excitation within a single-material 


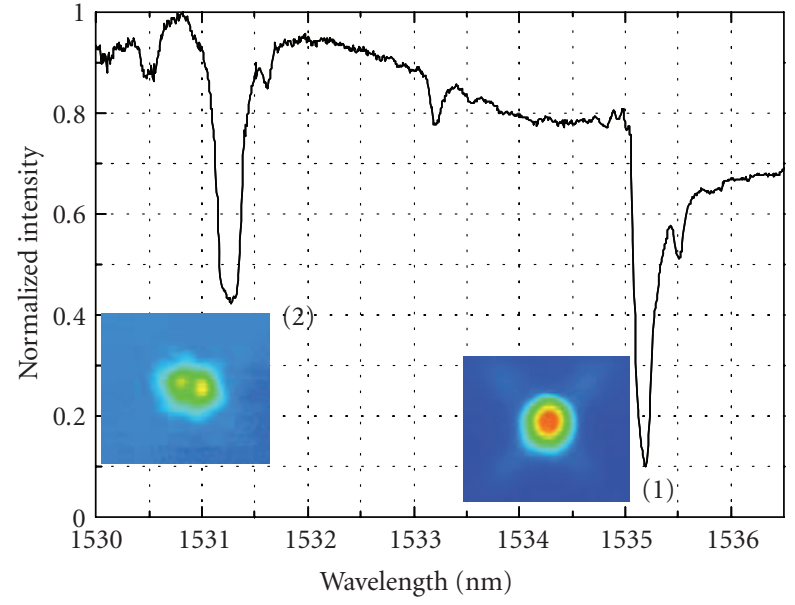

Figure 18: Transmission spectrum of a two-photon written Bragg gratings within a two-mode, 4-ring photonic crystal fibre. (1) fundamental mode; (2) higher order mode and the dip in the centre is some cross coupling between the two arises from some asymmetry in transverse profile of the induced index change [76].

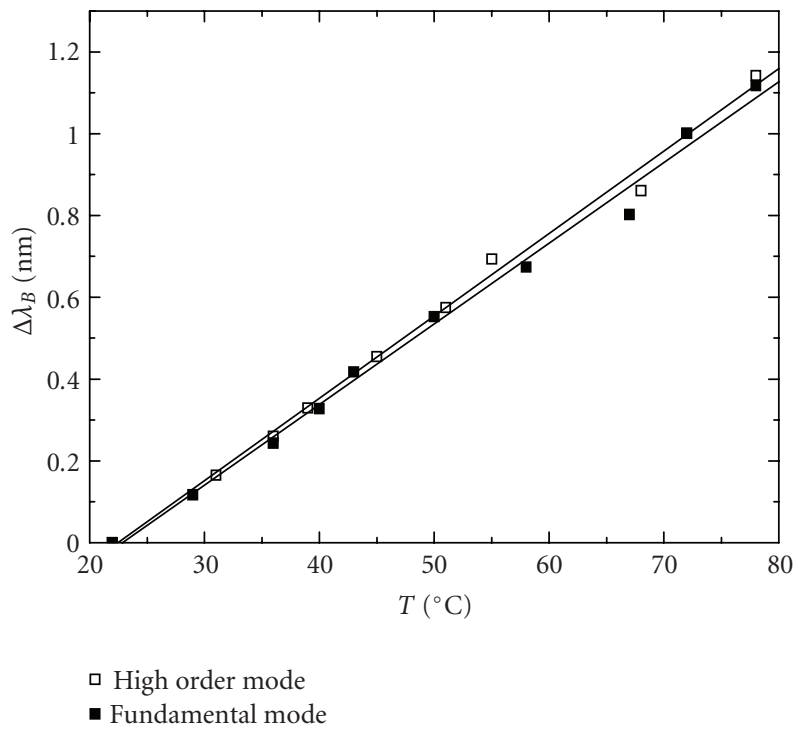

Figure 19: Temperature dependence of the two modes of the fibre described in Figure 18 [74, 76].

all-silica Fresnel fibre (Figure 1(c)) and a similar singlematerial, single-mode, all-silica 4-ring photonic crystal fibre (Figure 1(a)) as a function of temperature. Below $100^{\circ} \mathrm{C}$ the Fresnel fibre has a slightly higher temperature dependence, or effective thermooptic coefficient, $\left(\left(1 / n_{\text {eff } 1}\right)\left(\partial n_{\text {effl }} / \partial T\right)=\right.$ $7.8 \times 10^{-6} /{ }^{\circ} \mathrm{C}^{-1}$ compared to the photonic crystal fibre of $6.5 \times 10^{-6} /{ }^{\circ} \mathrm{C}$, although both are very close to that of pure silica $\sim 6 \times 10^{-6} /{ }^{\circ} \mathrm{C}[79]$. What is interesting to observe, is that unlike the previous results for the step-index $\mathrm{Er}^{3+}$ doped aluminosilicate step-index core photonic crystal fibre, there is a quadratic dependence on this effective thermooptic coefficient, which is particularly pronounced for the Fresnel fibre. This is directly attributable to mode field confinement

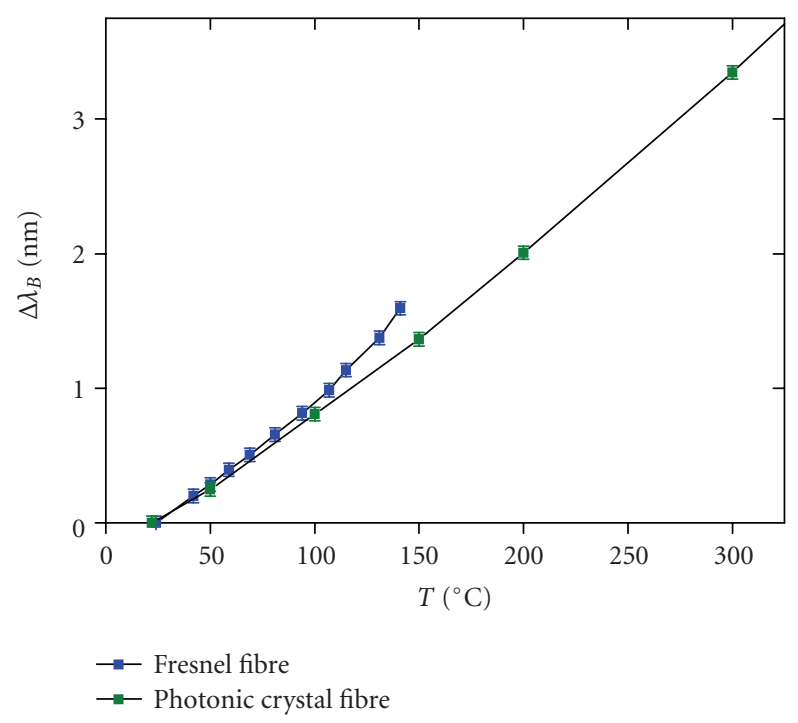

Figure 20: Temperature dependence of the fundamental mode within two types of structured optical fibre [15].

by the air holes as the index of the glass changes and, hence, great care must be taken when interpreting the temperature dependence of the structured optical fibres since this is not solely a material-dependent parameter as can be assumed in the case of conventional step-index fibres. The apparent observation of a sharp transition within the Fresnel fibre may characterize a cutoff condition for propagation which is especially sensitive in the Fresnel fibre given the larger dependence on diffractive propagation.

Clearly, the illustrated results reflect overall huge scope to adjust both the fibre design and fibre parameters and control properties such as the temperature dependence. This can be applied to enhance various configurations of thermally tunable devices using structured fibres, active or passive, and gratings, for example. For sensing applications, this control over the temperature dependence can be used to help separate the difficult corelationship between temperature and strain.

This example illustrates the flexibility in tailoring the properties of single-material fibres. Such fibres have other advantages-for example, given that the index contrast with air is sufficiently high, properties such as form birefringence can be readily introduced into these fibres. Form birefringence within a single-material fibre is free of the temperature problem associated with two materials of different thermal expansion coefficient-fibre that has a zero temperature dependence of birefringence from -20 to $+800^{\circ} \mathrm{C}$ has been demonstrated [80]. These results were extended to a spun version of this fibre [81], which has important applications, amongst many, to electric field sensors and gyroscopes.

\subsubsection{Strain Dependence}

In contrast to the temperature dependencies, there is a difference between the fundamental mode and the higher-order mode of the previous $\mathrm{Er}^{3+}$-doped aluminosilicate photonic 


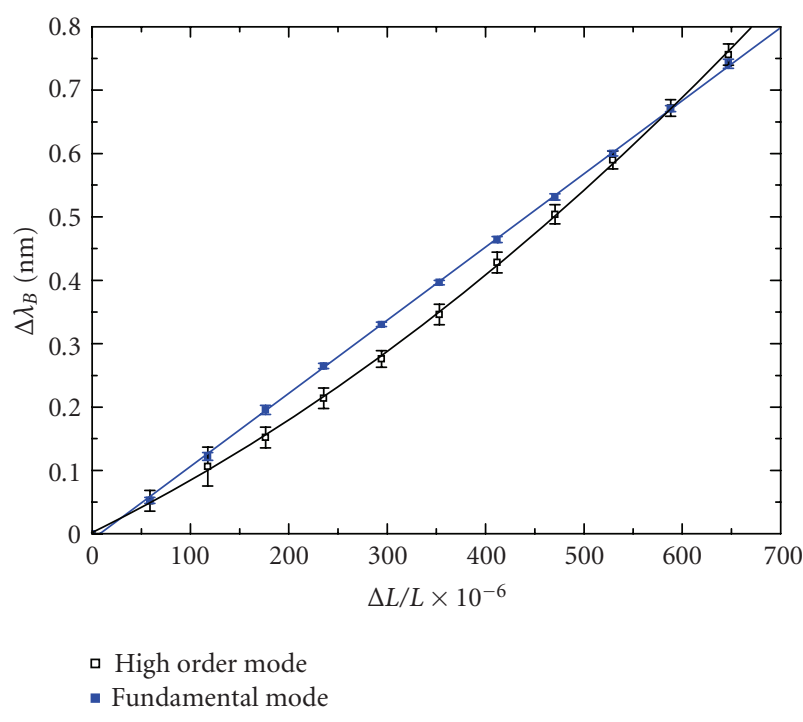

FIGURE 21: Wavelength shift versus applied longitudinal strain on the $\mathrm{Er}^{3+}$ doped, aluminosilicate dual mode photonic crystal fibre fibre. A quadratic dependence is observed for the higher order mode $[74,76]$.

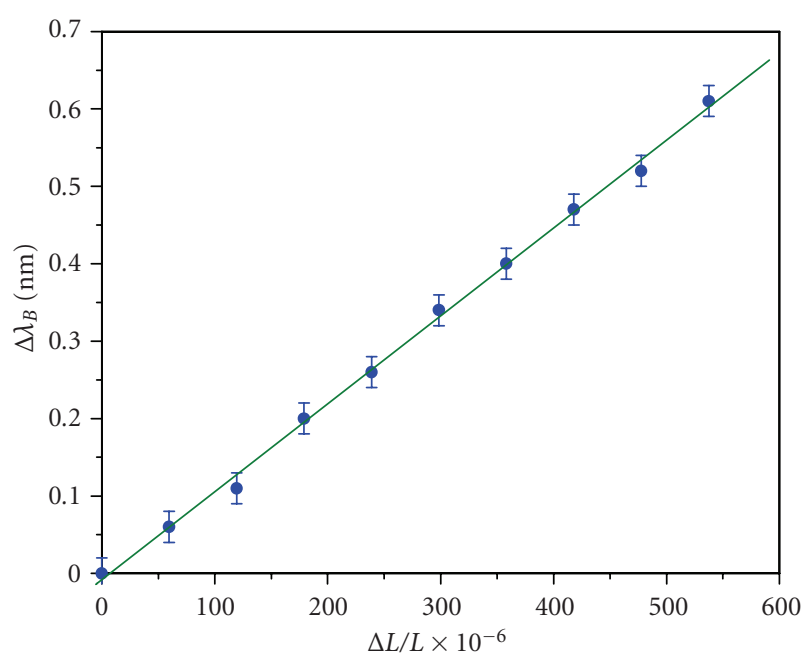

FIGURE 22: Wavelength shift versus applied longitudinal strain on the Fresnel fibre. No higher order mode is observed in this fibre [15].

crystal fibre. The observed shift with applied strain in the Bragg wavelength corresponding to the fundamental mode (Figure 21) has a linear behavior $\left(\partial \lambda_{\mathrm{B} 1} / \partial \varepsilon=1.2 \mathrm{pm} / \mu \varepsilon\right)$. However, the shorter Bragg wavelength, corresponding to the high-order mode, has nonlinear behavior described by a quadratic dependence. This arises from the compressive stress that is generated by the applied tensile strain (which can be estimated via Poisson's relations). Unlike conventional fibers, the higher-order leaky modes (in particular) of a photonic crystal fiber are sensitive to changes in stress between the holes and both the hole size and shape as well as the ratio of the hole diameter over the hole pitch, $d / \Lambda$ [82]. Since the holes are not centered, the compression of the first ring, in part arising as the solid core resists the compressive force, leads to an increase in $d / \Lambda$ with positive strain (applied tension). This problem is a well-known one that also contributes to hole deformation of the first ring during fiber fabrication if not addressed [83]. By increasing $d / \Lambda$, the modal confinement loss decreases [82], leading to improved confinement of the mode-this then alters the respective fractions of power, $\eta$, in the center core and in the silica ring and cladding. Since the effective refractive index is dependent on this fraction, there is an additional shift in the Bragg wavelength $\left(\lambda_{\mathrm{B}}=2 n_{\text {eff }} d\right)$ that deviates the curve from linearity. Given that the changes are based on circular confinement and therefore mode area, to first approximation a quadratic dependence $\left(\lambda \propto \mathrm{a} \varepsilon+\mathrm{b}^{2}\right)$ is expected and observed [76]. The strainoptic coefficient, therefore, reduces to $\partial \lambda_{\mathrm{B} 1} / \partial \varepsilon=\mathrm{a}+2 \mathrm{~b} \varepsilon$. Further, the grating strength is also dependent on this fraction $\left[\kappa=\tanh ^{2}(\pi \Delta v L \eta / \lambda)\right]$ and we expect to observe a change in grating strength as a function of strain. The air structure itself becomes integral to defining the material properties and in this context is indistinguishable from a composite material. Therefore, the whole medium can be considered a unique super-structured material (SSM) with properties that can be tailored by tailoring the refractive index in a way analogous to tailoring the atomic distribution of constituents and their fractions in composite systems.

In contrast to this fibre, and despite the large interaction of the sensitive fundamental diffractive mode with the structure given its reduced confinement and the absence of an insulating step-index core, the Fresnel fibre has only a linear dependence mode with strain, as shown in Figure 22 [15]. This is explained by the very low-air fraction and the irregular arrangement of holes that prevent a sponge-like structure susceptible to compressive effects.

The most obvious application of this work is to enable a simple distinction between strain and temperature from the perspective of controlling strain instead of temperature. The ability to remove, or unravel, the strain contribution is a key problem in fibre sensing with gratings generally and structured optical fibres offer a unique pathway to resolving this.

\subsection{Dual-Mode Operation for Sensing}

Above, we described the dual-mode operation of a structured fibre grating which effectively allows the fundamental mode to be the reference arm of a potential sensor based on such fibre whilst the higher-order mode is the probe arm. In this way, the temperature and strain can be separated out without additional fibre sensors being required. Recently, a much more sensitive version of such a device was demonstrated using a dual-mode DFB fibre laser inscribed directly into the same fibre [18].

\subsection{Diffraction from a Photonic Crystal Fibre}

We described above solid-core photonic crystal fibres by an analogous effective step-index fibre but noted that this 
approximation, used by many authors (see, e.g., [2] and the references therein), is generally not valid for higher-order modes. In fact, the effective core and cladding of this stepindex approximation can be highly sensitive to the extent of the modal field into the cladding. As a consequence of the high-core-cladding index contrast, the contribution to mode propagation from, for example, a periodic arrangement of holes is negligible when the wavelength of light is larger than the bridge thickness between the holes but smaller than the core diameter. On the other hand, for less confined modes or when the wavelength of light is commensurate or smaller than the interstitial hole spacing, as well as the core size itself, a periodic lattice provides phase conditions that allow coherent scattering of light and therefore diffractive confinement [84]. This resonant phenomenon has been observed in bending loss tests, where light leaks out from the core and is launched into the cladding, generating a short wavelength cutoff in the fibre transmission band [85]. This short wavelength cutoff is sensitive to the perturbations applied to the fibre as well as the index of the material within the hole. In fact, such regular coupling of light between the interstitial regions of a regular lattice-structured fibre plays a key role in high-bend loss characteristic of such fibres [86]. The best way to prevent this is, therefore, to remove the crystalline regularity of this lattice thereby spoiling the coupling —zero bend loss has been demonstrated using a chirped Fresnel fibre (or fractal fibre) [86] which in turn served as the basis for ideal tapered structured fibre for efficient metal free SNOM [87].

It has been proposed that these higher-order diffraction effects related to the lattice periodicity can even be used to advantage in novel sensors [25]. In fact, the corrugations associated with densification gratings produce the necessary coupling to the lattice through scattering. The condition for the wavelength dependence of this scattering will also be sensitive to what is in the air channels - in effect a refractive index diagnostic tool can be demonstrated. The refractive index of ice was measured this way, confirming how a simple Bragg condition suffices to describe the processes at short wavelengths. Therefore, the refractive index of the material within the holes, $n_{h}$, is given by [25]

$$
n_{h}=\frac{n_{\text {core }} \cos \left[\sin ^{-1}(\lambda m / 2 n \Lambda)\right]-x_{\mathrm{SiO}_{2}} n_{\mathrm{SiO}_{2}}}{x_{h}},
$$

where $n_{\text {cladding }}=x_{h} n_{h}+x_{\mathrm{SiO}_{2}} n_{\mathrm{SiO}_{2}}, x_{\mathrm{SiO}_{2}}$ and $x_{h}$ are the fractions of silica and holes that make up the cladding, $m$ is the grating order, $\Lambda$ is the lattice pitch, and $n_{\mathrm{SiO}_{2}}$ the index of $\mathrm{SiO}_{2}$. Figure 23 shows the calculated refractive index of water determined from the transmission band edge shift in the visible to longer wavelengths [25] as a function of temperature. Freezing is observed to occur at a lower temperature than zero, $\sim-3^{\circ} \mathrm{C}$, consistent with very high pressures induced within the microchannel. To lower the m.p. of ice by such an amount requires a local pressure $>30 \mathrm{Mpa}$ [88], giving an indication of the high-effective pressures that can be generated within micro- and nanocapillaries of ice 1 hour, which has $\sim 9 \%$ volume increase from the liquid state. This has important implications for micro- and nanofluidics. The

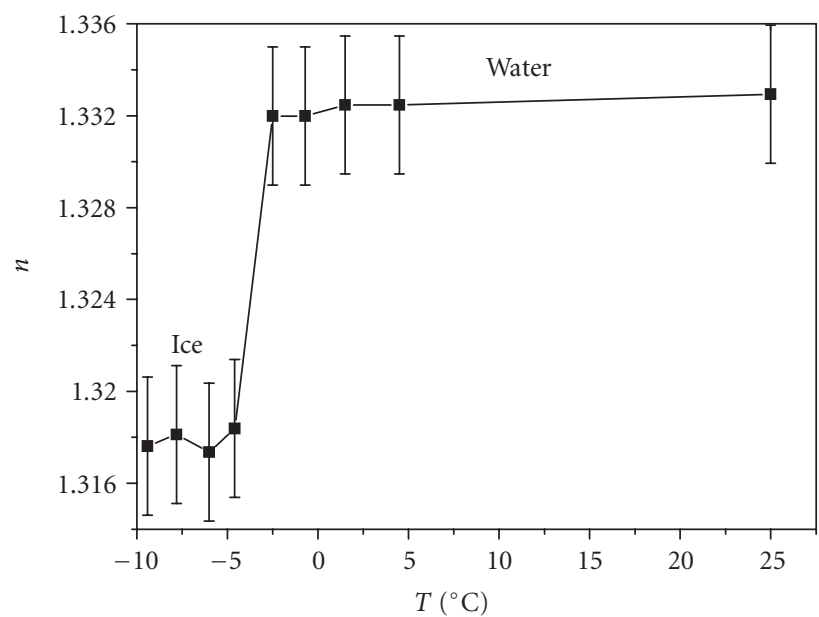

Figure 23: Refractive index measurement of the water and ice $1 \mathrm{H}$ before, during, and after freezing [25].

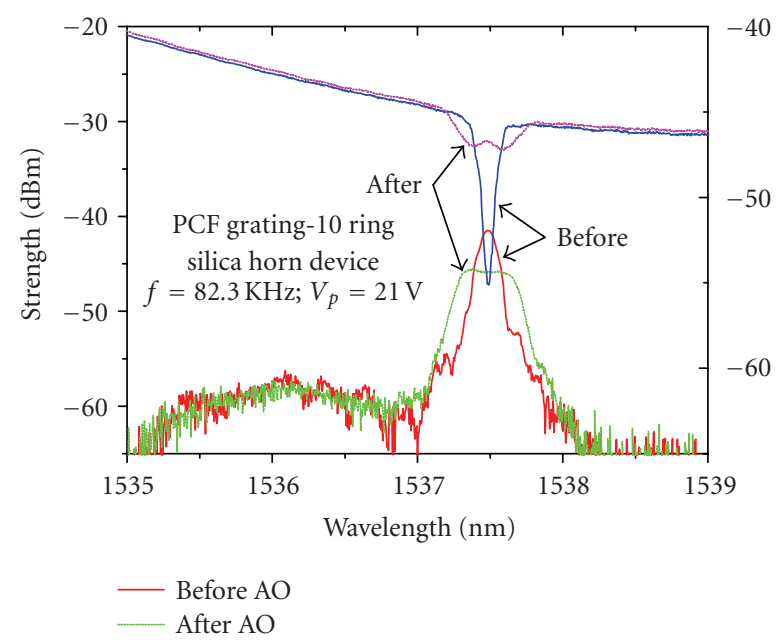

FIGURE 24: Transmission and reflection spectra of a uniform Bragg grating in a germanosilicate-doped core photonic crystal fibre with and without an applied acoustic wave with frequency $\sim 82.3 \mathrm{kHz}$. At this frequency, a central phase shift is introduced and the band gap broadens. This change was sufficient to generate $>10 \mathrm{~dB}$ signal contrast when switching a tuneable laser on and off [89].

calculated refractive index for the frozen ice is in agreement within error with that of ice 1 hour as expected.

\subsection{Acoustic Tuning of PCF Gratings}

Given the control over the cladding parameters, including the size and positioning of the holes, within a structured optical fibre another property which may be expected to differ is the generation and propagation of acoustic waves within the fibre. This has particular important applications within acoustic-based pressure and hydrophone sensors, including DFB fibre laser arrays in underwater detection of seismic waves, enemy submarine and naval craft, and more. It is, therefore, of note that the first recent reports of acoustic waves in photonic crystal fibre involved Bragg 
gratings inscribed into the fibres for these applications [8991]. A tuneable Moire grating was demonstrated for the first time [89] by having an acoustic standing wave established over the Bragg grating which leads to bandwidth broadening and a phase-shifted structure as shown in Figure 22. Further work is underway to explore the potential of structured fibres in enhancing and controlling these properties.

\section{Conclusions}

Considerable work has already been carried out in the inscription of Bragg gratings and other structures within structured optical fibres. Both positive and negative index gratings in Type I regime (below the damage threshold [22]) are easily demonstrated and unsurprising within structure fibres containing standard photosensitive cores. However, the control and refinement of this writing process is considerably more difficult as a result of the rotational variance of the optical fibre. Progress in this direction has led to a considerable improvement in the reliable inscription of gratings. More challenging, but in many ways far more important, has been the inscription of higher-exponent photon gratings, including densification and damage gratings, that do not rely on accessing defects so that much of the advantages of structured fibres, including singlematerial fibres with no temperature dependence arising from differences in expansion coefficients. Some of the distinct advantages of structured fibres have been argued from the perspective of the grating itself. Gratings act as both a critical diagnostic interferometer and as an important spectrally selective component to further extend these advantages in much the same way gratings have, and continue, to extend conventional optical fibre. Applications are liberal spanning from telecommunications, lasers, sensors, and diagnostics.

\section{Acknowledgments}

The authors would like to acknowledge various students, staff, and colleagues, both local (Andrew Michie and Mattias Aslund from IPL, Sydney University, Graham Marshall, Nem Jovanovic et al. from Macquarie University; Shane Huntington et al. from Melbourne University) and overseas (Henrick Sorenson, Hans Deyerl, Martin Kristensen et al. from Research Centre COM, Danish Technical University; Matthieu Lancry and Bertrand Poumellec, Universide de Paris Sude, France), who have contributed over recent years to some aspects of the described work. Funding for this work came from the Australian Research Council and the Department of Education, Science and Training (DEST), Australia. Cicero Martelli acknowledges a CAPES, Brazil Student scholarship, and Somnath Bandyopadhyay acknowledges a Raman Research Fellowship of CSIR, India for his stay in Australia.

\section{References}

[1] P. Kaiser and H. W. Astle, "Low-loss single-material fibers made from pure fused silica," Bell System Technical Journal, vol. 53, no. 6, pp. 1021-1039, 1974.
[2] A. Bjarklev, J. Broeng, and A. S. Bjarklev, Photonic Crystal Fibres, Kluwer Academic Publishers, Boston, Mass, USA, 2003.

[3] J. Canning, "Fresnel optics inside optical fibres," in Photonics Research Developments, chapter 4, Nova Science, Huntington, NY, USA, 2008.

[4] H. R. Sørensen, J. Canning, J. Lægsgaard, K. Hansen, and P. Varming, "Liquid filling of photonic crystal fibres for grating writing," Optics Communications, vol. 270, no. 2, pp. 207-210, 2007.

[5] J. Canning, S. K. Lim, T. K. Yip, and C. Martelli, "New device functionality within structured optical fibres by selective filling," in Proceedings of the Joint Conference on OptoElectronics and Communications Conference and the Australian Conference on Optical Fibre Technology (ACOFT/OECC '08), Sydney, Australia, July 2008.

[6] C. Martelli, J. Canning, J. R. Reimers, M. Sintic, D. Stocks, and M. J. Crossley, "Evanescent field spectroscopy using structured optical fibres: detection of charge-transfer at the porphyrinsilica interface," submitted to Journal of the American Chemical Society.

[7] B. J. Eggleton, P. S. Westbrook, R. S. Windeler, S. Spälter, and T. A. Strasser, "Grating resonances in air-silica microstructured optical fibers," Optics Letters, vol. 24, no. 21, pp. 1460-1462, 1999.

[8] H. R. Sørensen, J. Canning, J. Lægsgaard, K. Hansen, and P. Varming, "Liquid filling of photoniccrystal fibres for grating writing," Optics Communications, vol. 270, no. 2, pp. 207-210, 2007.

[9] V. Beugin, L. Bigot, P. Niay, et al., "Efficient Bragg gratings in phosphosilicate and germanosilicate photonic crystal fiber," Applied Optics, vol. 45, no. 32, pp. 8186-8193, 2006.

[10] L. B. Fu, G. D. Marshall, J. A. Bolger, et al., "Femtosecond laser writing Bragg gratings in pure silica photonic crystal fibres," Electronics Letters, vol. 41, no. 11, pp. 638-640, 2005.

[11] M. C. Phan Huy, G. Laffont, Y. Frignac, et al., "Fibre Bragg grating photowriting in microstructured optical fibres for refractive index measurement," Measurement Science and Technology, vol. 17, no. 5, pp. 992-997, 2006.

[12] G. Violakis and S. Pissadakis, "Improved efficiency Bragg grating inscription in a commercial solid core microstructured optical fiber," in Proceedings of the 9th International Conference on Transparent Optical Networks (ICTON '07), vol. 2, pp. 217220, Rome, Italy, July 2007.

[13] J. Albert, M. Fokine, and W. Margulis, "Grating formation in pure silica-core fibers," Optics Letters, vol. 27, no. 10, pp. 809$811,2002$.

[14] N. Groothoff, J. Canning, E. Buckley, K. Lyttikainen, and J. Zagari, "Bragg gratings in air-silica structured fibers," Optics Letters, vol. 28, no. 4, pp. 233-235, 2003.

[15] N. Groothoff, C. Martelli, J. Canning, and K. Lyytikainen, "Fibre Bragg grating in Fresnel fibre with temperature and strain characterisation," in Proceedings of the 30th Australian Conference on Optical Fibre Technology (ACOFT '05), Sydney, Australia, July 2005.

[16] J. Canning, N. Groothoff, E. Buckley, T. Ryan, K. Lyytikainen, and J. Digweed, "All-fibre photonic crystal distributed Bragg reflector (PC-DBR) fibre laser," Optics Express, vol. 11, no. 17, pp. 1995-2000, 2003.

[17] N. Groothoff, J. Canning, T. Ryan, K. Lyytikainen, and H. Inglis, "Distributed feedback photonic crystal fibre (DFBPCF) laser," Optics Express, vol. 13, no. 8, pp. 2924-2930, 2005.

[18] N. Groothoff, C. Martelli, and J. Canning, "A dual wavelength distributed-feedback fiber laser," Journal of Applied Physics, vol. 103, no. 1, Article ID 013101, 6 pages, 2008. 
[19] J. Canning, S. D. Jackson, M. L. Åslund, N. Groothoff, B. Ashton, and K. Lyytikainen, "Air-clad fibre laser with internal Bragg grating," Electronics Letters, vol. 41, no. 20, pp. 1103 1104, 2005.

[20] N. Groothoff, J. Canning, N. Jovanovic, G. D. Marshall, and M. J. Whitford, "Gratings in large diameter air-clad optical fibre using a femtosecond laser," in Proceedings of the OSA Topical Meeting: Bragg Gratings, Photosensitivity and Poling (BGPP '07), Quebec City, Canada, September 2007.

[21] S. J. Mihailov, D. Grobnic, H. Ding, C. W. Smelser, and J. Broeng, "Femtosecond IR laser fabrication of Bragg gratings in photonic crystal fibers and tapers," IEEE Photonics Technology Letters, vol. 18, no. 17, pp. 1837-1839, 2006.

[22] J. Canning, "Fibre gratings and devices," in Lasers and Photonics Reviews, John Wiley \& Sons, New York, NY, USA, 2008.

[23] C. Martelli, J. Canning, B. Gibson, and S. Huntington, "Cryptography based on coherent scattering of light," in Proceedings of the Joint International Conference on Optical Internet and the 32nd Australian Conference on Optical Fibre Technology (COIN-ACOFT '07), pp. 1-3, Melbourne, Australia, June 2007.

[24] R. C. Gauthier, "Photonic quasi-crystals: a review," in Proceedings of the Progress in Electromagnetics Research Symposium (PIERS '08), p. 151, Hangzhou, China, March 2008.

[25] C. Martelli, J. Canning, M. Kristensen, and N. Groothoff, "Refractive index measurement within a photonic crystal fibre based on short wavelength diffraction," Sensors, vol. 7, no. 11, pp. 2492-2498, 2007.

[26] J. De Iuliis, "Modelling the angular dependence on Bragg grating writing in photonic crystal fibre," Student Practical Optical Design Report, University of Newcastle, 2006.

[27] G. D. Marshall, D. J. Kan, A. A. Asatryan, L. C. Botten, and M. J. Withford, "Transverse coupling to the core of a photonic crystal fiber: the photo-inscription of gratings," Optics Express, vol. 15, no. 12, pp. 7876-7887, 2007.

[28] J. Canning, "Gratings and grating devices in structured fibres using $193 \mathrm{~nm}$ from an ArF laser," in Proceedings of the OSA Topical Meeting: Bragg Gratings, Photosensitivity and Poling (BGPP '07), Quebec City, Canada, September 2007.

[29] J. L. Holdsworth, J. Canning, and C. Dewhurst, "Rotational dependence of laser light accessing photonic crystal fibre cores from the side," in Proceedings of the 19th International Conference on Optical Fibre Sensors, D. Sampson, S. Collins, K. Oh, and R. Yamauchi, Eds., vol. 7004 of Proceedings of SPIE, pp. 1-4, Perth, Australia, April 2008.

[30] J. Holdsworth, S. Bandyopadhyay, J. Canning, M. Stevenson, and J. de Iuliis, "Transversely illuminating the core of photonic crystal fibres," in Proceedings of the Joint Conference of the Opto-Electronics and Communications Conference and the Australian Conference on Optical Fibre Technology (OECC/ACOFT '08), Sydney, Australia, July 2008.

[31] J. Holdsworth, K. Cook, J. Canning, S. Bandyopadhyay, and M. Stevenson, "Rotationally variant grating writing in photonic crystal fibres," submitted to Optics Express.

[32] A. P. Mosk, "Focussing of light by disordered metamaterials," in Proceedings of the Progress in Electromagnetics Research Symposium (PIERS '08), p. 741, Hangzhou, China, March 2008.

[33] K. Cook, A. Pohl, and J. Canning, "Positive and negative index gratings in 10-ring photonic crystal fibres with germanosilicate cores using $193 \mathrm{~nm}$," in Proceedings of the
Joint Conference of the Opto-Electronics and Communications Conference and the Australian Conference on Optical Fibre Technology (OECC/ACOFT '08), Sydney, Australia, July 2008.

[34] K. Cook, A. Pohl, and J. Canning, "Type IIA gratings in hydrogen-free 10-ring photonic crystal fibre with germanosilicate core," submitted to Optics Letters.

[35] N. Groothoff and J. Canning, "Enhanced type IIA gratings for high-temperature operation," Optics Letters, vol. 29, no. 20, pp. 2360-2362, 2004.

[36] J. Canning, K. Sommer, M. Englund, and S. Huntington, "Direct evidence of two types of UV-induced glass changes in silicate-based optical fibers," Advanced Materials, vol. 13, no. 12-13, pp. 970-973, 2001.

[37] H. Sorensen, J. B. Jensen, F. Bruyere, and K. P. Hansen, "Practical hydrogen loading of air silica fibers," in Bragg Gratings Photosensitivity and Poling in Glass Waveguides, Trends in Optics and Photonics Series, Optical Society of America, Washington, DC, USA, 2005.

[38] D. Káčik, I. Turek, I. Martinček, J. Canning, N. A. Issa, and K. Lyytikäinen, "Intermodal interference in a photonic crystal fibre," Optics Express, vol. 12, no. 15, pp. 3465-3470, 2004.

[39] A. Pohl, K. Cook, and J. Canning, "Bragg grating writing in $\mathrm{H}_{2}$-loaded many-layered PCF without liquid filling of the holes," in Proceedings of the Joint Conference of the OptoElectronics and Communications Conference and the Australian Conference on Optical Fibre Technology (OECC/ACOFT '08), Sydney, Australia, July 2008.

[40] M. Janos and J. Canning, "Permanent and transient resonances thermally induced in optical fibre Bragg gratings," Electronics Letters, vol. 31, no. 12, pp. 1007-1009, 1995.

[41] A. Canagasabey, J. Canning, J. Mills, D. P. Banks, and M. Ibsen, "Fibre Bragg grating fabrication in germanosilicate fibres with $244 \mathrm{~nm}$ femtosecond laser light," in Proceedings of the 2nd Pacific International Conference on Applications of Lasers and Optics (PICALO '06), Melbourne, Australia, April 2006.

[42] A. Wootton, B. Thomas, and P. Harrowell, "Radiation-induced densification in amorphous silica: a computer simulation study," Journal of Chemical Physics, vol. 115, no. 7, pp. 33363341, 2001.

[43] C. Martelli, J. Canning, M. Kristensen, and N. Groothoff, "Impact of water and ice $1 \mathrm{~h}$ formation in a photonic crystal fiber grating," Optics Letters, vol. 31, no. 6, pp. 706-708, 2006.

[44] W. Primak, L. H. Fuchs, and P. Day, "Radiation damage in insulators,” Physical Review, vol. 92, no. 4, pp. 1064-1065, 1953.

[45] W. Primak and H. Szymanski, "Radiation damage in vitreous silica: annealing of the density changes," Physical Review, vol. 101, no. 4, pp. 1268-1271, 1956.

[46] W. Primak and R. Kampwirth, "The radiation compaction of vitreous silica," Journal of Applied Physics, vol. 39, no. 12, pp. 5651-5658, 1968.

[47] C. Fiori and R. A. B. Devine, "Evidence for a wide continuum of polymorphs in $a-\mathrm{SiO}_{2}$," Physical Review B, vol. 33, no. 4, pp. 2972-2974, 1986.

[48] M. Lancry and B. Poumellec, "Multiphoton absorption processes \& UV laser processing of silica-based materials," in Proceedings of the 1st International Workshop on Multiphoton Processes in Glass and Glassy Materials, J. Canning, Ed., Sydney, Australia, December 2006.

[49] N. Groothoff, M. Lancry, B. Poumellec, and J. Canning, "193 nm photolytic mechanism in Er/Al doped silica," in Proceedings of the Joint Conference on Opto-Electronics and Communications Conference and the Australian Conference 
on Optical Fibre Technology (ACOFT/OECC '08), Sydney, Australia, July 2008.

[50] M. Lancry, N. Groothoff, B. Poumellec, and J. Canning, "Twophoton grating writing in Er/Al doped preform plates using $193 \mathrm{~nm}$ laser light," In preparation.

[51] A. Dragomir, D. N. Nikogosyan, K. A. Zagorulko, P. G. Kryukov, and E. M. Dianov, "Inscription of fiber Bragg gratings by ultraviolet femtosecond radiation," Optics Letters, vol. 28, no. 22, pp. 2171-2173, 2003.

[52] S. J. Mihailov, C. W. Smelser, P. Lu, et al., "Fiber Bragg gratings made with a phase mask and 800 -nm femtosecond radiation," Optics Letters, vol. 28, no. 12, pp. 995-997, 2003.

[53] K. A. Zagorulko, P. G. Kryukov, Yu. V. Larionov, et al., "Fabrication of fiber Bragg gratings with $267 \mathrm{~nm}$ femtosecond radiation," Optics Express, vol. 12, no. 24, pp. 5996-6001, 2004.

[54] A. Martinez, M. Dubov, I. Khrushchev, and I. Bennion, "Direct writing of fibre Bragg gratings by femtosecond laser," Electronics Letters, vol. 40, no. 19, pp. 1170-1172, 2004.

[55] C. W. Smelser, S. J. Mihailov, D. Grobnic, et al., "Multiplebeam interference patterns in optical fiber generated with ultrafast pulses and a phase mask," Optics Letters, vol. 29, no. 13, pp. 1458-1460, 2004.

[56] C. W. Smelser, S. J. Mihailov, and D. Grobnic, "Hydrogen loading for fiber grating writing with a femtosecond laser and a phase mask," Optics Letters, vol. 29, no. 18, pp. 2127-2129, 2004.

[57] C. W. Smelser, S. J. Mihailov, and D. Grobnic, "Formation of type I-IR and type II-IR gratings with an ultrafast IR laser and a phase mask," Optics Express, vol. 13, no. 14, pp. 5377-5386, 2005.

[58] in Proceedings of the 1st International Workshop on Multiphoton Processes in Glass and Glassy Materials, J. Canning, Ed., Sydney, Australia, December 2006.

[59] C. G. Askins, T.-E. Tsai, G. M. Williams, M. A. Putnam, M. Bashkansky, and E. J. Friebele, "Fiber Bragg reflectors prepared by a single excimer pulse," Optics Letters, vol. 17, no. 11, pp. 833-835, 1992.

[60] M. L. Åslund, N. Jovanovic, N. Groothoff, et al., "Large diffractive scattering losses in the visible region produced by femtosecond laser written Bragg gratings," in Proceedings of the Australian Conference on Optical Fibre Technology \& Opto-Electronics and Communications Conference (ACOFT/OECC '08), Sydney, Australia, July 2008.

[61] M. L. Åslund, N. Jovanovic, N. Groothoff, et al., "Optical loss mechanisms in femtosecond laser-written point-by-point fibre Bragg gratings," Optics Express, vol. 16, no. 18, pp. 1424814254, 2008.

[62] N. Jovanovic, M. L. Åslund, A. Fuerbach, S. D. Jackson, G. D. Marshall, and M. J. Withford, "Narrow linewidth, $100 \mathrm{~W}$ $\mathrm{cW} \mathrm{Yb}^{3+}$-doped silica fiber laser with a point-by-point Bragg grating inscribed directly into the active core," Optics Letters, vol. 32, no. 19, pp. 2804-2806, 2007.

[63] M. L. Åslund, S. D. Jackson, J. Canning, N. Groothoff, B. Ashton, and K. Lyytikainen, "High power $\mathrm{Yb}^{3+}$ doped airclad fibre laser using a Bragg grating written into the active medium," in Proceedings of the 7th Australian Conference on Optics, Lasers and Spectroscopy (ACOLS '05), Roturua, New Zealand, December 2005, paper WeA2.

[64] M. L. Åslund, private communication, unpublished work, 2007.

[65] J. Arkwright, G. Atkins, Z. Brodzeli, et al., "All optical switching in rare-earth doped fibers," in Proceedings of the Pacific Rim Conference on Lasers and Electro-Optics (CLEO/Pacific Rim '97), p. 265, Chiba, Japan, July 1997, paper FH1.
[66] E. N. Glezer and E. Mazur, "Ultrafast-laser driven microexplosions in transparent materials," Applied Physics Letters, vol. 71, no. 7, pp. 882-884, 1997.

[67] K. Sugioka, Y. Cheng, and K. Midorikawa, "Threedimensional micromachining of glass using femtosecond laser for lab-on-a-chip device manufacture," Applied Physics A, vol. 81, no. 1, pp. 1-10, 2005.

[68] J. W. Chan, T. R. Huser, S. H. Risbud, and D. M. Krol, "Modification of the fused silica glass network associated with waveguide fabrication using femtosecond laser pulses," Applied Physics A, vol. 76, no. 3, pp. 367-372, 2003.

[69] S. A. Slattery, D. N. Nikogosyan, and G. Brambilla, "Fiber Bragg grating inscription by high-intensity femtosecond UV laser light: comparison with other existing methods of fabrication," Journal of the Optical Society of America B, vol. 22, no. 2, pp. 354-361, 2005.

[70] L. Shah, A. Arai, S. Eaton, and P. Herman, "Waveguide writing in fused silica with a femtosecond fiber laser at $522 \mathrm{~nm}$ and $1 \mathrm{MHz}$ repetition rate," Optics Express, vol. 13, no. 6, pp. 1999 2006, 2005.

[71] K. Digweed-Lyytikainen, C. A. De Francisco, D. Spadoti, et al., "Photonic crystal optical fibers for dispersion compensation and Raman amplification: design and experiment," Microwave and Optical Technology Letters, vol. 49, no. 4, pp. 872-874, 2007.

[72] M. L. Åslund, J. Canning, S. D. Jackson, A. Teixeira, and K. Lyytikäinen, "Diffraction in air-clad fibres," Optics Express, vol. 13, no. 14, pp. 5227-5233, 2005.

[73] M. L. Åslund, S. D. Jackson, J. Canning, A. Teixeira, and K. Lyytikäinen-Digweed, "The influence of skew rays on angular losses in air-clad fibres," Optics Communications, vol. 262, no. 1, pp. 77-81, 2006.

[74] C. Martelli, J. Canning, N. Groothoff, and K. Lyytikainen, "Strain and temperature characterization of photonic crystal fiber Bragg gratings," Optics Letters, vol. 30, no. 14, pp. 17851787, 2005.

[75] D. Káčik, I. Turek, I. Martinček, J. Canning, N. A. Issa, and K. Lyytikäinen, "Intermodal interference in a photonic crystal fibre," Optics Express, vol. 12, no. 15, pp. 3465-3470, 2004.

[76] C. Martelli, J. Canning, N. Groothoff, and K. Lyttikainen, "Bragg gratings in photonic crystal fibers: strain and temperature chacterization," in 17th International Conference on Optical Fibre Sensors, M. Voet, R. Willsch, W. Ecke, J. Jones, and B. Culshaw, Eds., vol. 5855 of Proceedings of SPIE, pp. 302305, Bruges, Belgium, May 2005.

[77] M. Janos and J. Canning, "Permanent and transient resonances thermally induced in optical fibre Bragg gratings," Electronics Letters, vol. 31, no. 12, pp. 1007-1009, 1995.

[78] H. R. Sørensen, J. Canning, J. Lægsgaard, and K. Hansen, "Control of the wavelength dependent thermo-optic coefficients in structured fibres," Optics Express, vol. 14, no. 14, pp. 6428-6433, 2006.

[79] O. V. Mazurin, M. V. Streltsina, and T. P. Shvaikoshvaikovskaya, Eds., Handbook of Glass Data. Part A. Silica Glass and Binary Silicate Glasses, Elsevier, Amsterdam, The Netherlands, 1983.

[80] A. Michie, J. Canning, K. Lyytikäinen, M. L. Åslund, and J. Digweed, "Temperature independent highly birefringent photonic crystal fibre," Optics Express, vol. 12, no. 21, pp. 5160-5165, 2004.

[81] A. Michie, J. Canning, I. Bassett, et al., "Spun elliptically birefringent photonic crystal fibre," Optics Express, vol. 15, no. 4, pp. 1811-1816, 2007. 
[82] D. Ferrarini, L. Vincetti, M. Zoboli, A. Cucinotta, and S. Selleri, "Leakage properties of photonic crystal fibers," Optics Express, vol. 10, no. 23, pp. 1314-1319, 2002.

[83] K. Lyytikäinen, Control of complex structural geometry in optical fibre drawing, Ph.D. thesis, School of Physics and Interdisciplinary Photonics Laboratories, The University of Sydney, Sydney, Australia, 2004.

[84] J. Canning, "Diffraction-free mode generation and propagation in optical waveguides," Optics Communications, vol. 207, no. 1-6, pp. 35-39, 2002.

[85] D. Káčik, I. Turek, I. Martinček, J. Canning, and K. Lyytikainen, "The role of diffraction in influencing the short wavelength loss edge of photonic crystal fibres," in Proceedings of the 30th Australian Conference on Optical Fibre Technology (ACOFT '05), Sydney, Australia, July 2005.

[86] C. Martelli, J. Canning, B. Gibson, and S. Huntington, "Bend loss in structured optical fibres," Optics Express, vol. 15, no. 26, pp. 17639-17644, 2007.

[87] C. M. Rollinson, S. M. Orbons, S. T. Huntington, et al., "Near field characterisation of a nano-structured metamaterial with a metal-free optical fiber probe," submitted to Nano Letters.

[88] V. F. Petrenko and R. W. Whitworth, Physics of Ice, Oxford University Press, New York, NY, USA, 2002.

[89] A. A. P. Pohl, R. A. Oliveira, K. Cook, and J. Canning, "Modulation of a photonic crystal fibre Bragg grating induced by acoustical waves," submitted to Optics Express.

[90] A. A. P. Pohl, P. T. Neves Jr., R. A. Oliveira, M. Stevenson, N. Groothoff, and J. Canning, "Technique for estimating the tuning speed of fiber Bragg gratings," in 19th International Conference on Optical Fibre Sensors, vol. 7004 of Proceedings of SPIE, Perth, Australia, April 2008.

[91] R. A. Oliveira, P. de T. Neves Jr., J. M. Maia, A. de A. P. Pohl, and J. Canning, "Modulação da Refletividade de Redes de Bragg usando Interação Acusto-Óptica de Baixa Freqüência," in Proceedings of the 13 SBMO Brazilian Symposium on Microwave and Optoelectronics and 8th CBMag Brazilian Congress of Eletromagnetismo (MOMAG '08), pp. 396-398, Florianpolis, Brazil, September 2008. 